Homology, Homotopy and Applications, vol. 20(2), 2018, pp.11-40

\title{
L-HOMOLOGY ON BALL COMPLEXES AND PRODUCTS
}

\author{
SPIROS ADAMS-FLOROU AND TIBOR MACKO
}

\author{
(communicated by John R. Klein)
}

\begin{abstract}
We construct homology theories with coefficients in $L$-spectra on the category of ball complexes and we define products in this setting. We also obtain signatures of geometric situations in these homology groups and prove product formulae which we hope will clarify products used in the theory of the total surgery obstruction.
\end{abstract}

\section{Introduction}

Let $X$ be an $n$-dimensional geometric Poincaré complex, that means a finite $C W$ complex satisfying Poincaré duality. A fundamental question in topology of manifolds is to decide whether $X$ is homotopy equivalent to an $n$-dimensional topological manifold. Ranicki developed a systematic general theory for answering this question resulting in the definition of the total surgery obstruction $s(X) \in \mathbb{S}_{n}(X)=L_{n-1}\left(\Lambda_{*}^{c}(X)\right)$ which if $n \geqslant 5$ is zero if and only if the answer is yes, see [Ran79, Ran92, KMM13]. Here $\mathbb{S}_{n}(X)=L_{n-1}\left(\Lambda_{*}^{c}(X)\right)$ is the $L$-group of the algebraic bordism category of quadratic chain complexes over $X$ which are locally Poincaré and globally contractible and satisfy certain connectivity assumptions which we suppress. The theory can also be used in the relative setting when it answers the question of whether two manifold structures $f_{0}: M_{0} \stackrel{\simeq}{\rightarrow} X$ and $f_{1}: M_{1} \stackrel{\simeq}{\rightarrow} X$ are homeomorphic over $X$, that means whether they define the same element in the topological structure set $\mathcal{S}^{\text {TOP }}(X)$ in the sense of surgery theory. In fact, the theory results in a bijection $s: \mathcal{S}^{\mathrm{TOP}}(X) \rightarrow \mathbb{S}_{n+1}(X)=L_{n}\left(\Lambda_{*}^{c}(X)\right)$, see again [Ran79, Ran92, KMM13].

In the process of developing the theory the whole setup of geometric surgery and algebraic surgery is used. In particular, the set of normal invariants (alias degree one normal maps), denoted here $\mathcal{N}^{\mathrm{TOP}}(X)$, is used and a bijection is obtained

$$
\operatorname{qsign}_{X}: \mathcal{N}^{\mathrm{TOP}}(X) \rightarrow H_{n}\left(X ; \mathbf{L}_{\bullet}\langle 1\rangle\right) \cong L_{n}\left(\Lambda_{*}(X)\right) .
$$

Here $H_{n}(X ; \mathbf{L} \bullet\langle 1\rangle) \cong L_{n}\left(\Lambda_{*}(X)\right)$ is the homology of $X$ with respect to the 1 -connective cover of the quadratic $L$-theory spectrum L. [Ran92, Chapter 15] and as the isomorphism suggests it can be obtained as the $L$-group of the algebraic bordism category of quadratic chain complexes over $X$ which are locally Poincaré and again satisfy certain connectivity conditions which we suppress. The map (1.1) is called the quadratic signature over $X$, it is obtained by refining the quadratic construction of [Ran80b].

Received February 8, 2017, revised December 6, 2017; published on April 18, 2018.

2010 Mathematics Subject Classification: 57R65.

Key words and phrases: L-theory, surgery theory, ball complex.

Article available at http://dx.doi.org/10.4310/HHA.2018.v20.n2.a2

Copyright (C) 2018, International Press. Permission to copy for private use granted. 
In fact, the quadratic signature over $X$ provides us in the case that $X$ is an $n$ dimensional manifold such that $\pi=\pi_{1}(X)$ and $n \geqslant 5$ with an identification of the geometric and algebraic surgery exact sequences:

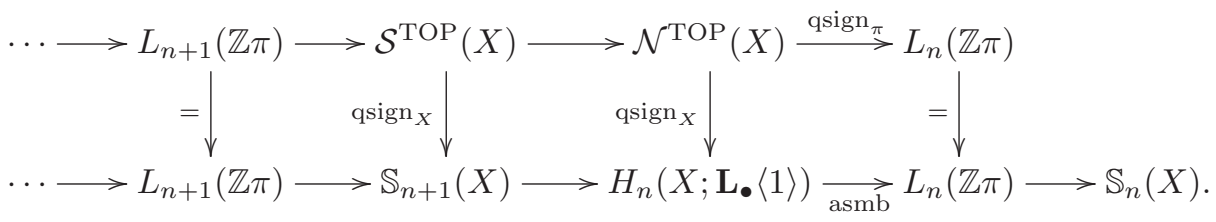

Here qsign $_{\pi}$ is the surgery obstruction map obtained via the above mentioned quadratic construction of [Ran80b] and asmb is the assembly map, see [Ran92, KMM13] for more details.

However, there are some deficiencies in the setup of [Ran79, Ran92, KMM13] when we are interested in its behavior with respect to products. Firstly recall that there is a well developed theory for products in $L$-groups of rings in [Ran80a, Section 8] and [Ran80b, Section 8] which includes product formulae for geometric situations. However, when considering the terms in the sequences above, we observe that the setup relies on choosing a simplicial complex model for $X$ and working with categories of modules and chain complexes over it. In [Ran92, Appendix B] it is described how to use a variant of a simplicial diagonal approximation to obtain certain products. However, from our point of view that description is not sufficient since we could not obtain product formulae for the map (1.1). More similar products appear in [Ran92, Chapter 21] and in the Appendix to [LR92] again referring to the simplicial diagonal approximation.

Recently Laures and McClure [LM14] used ball complexes instead of simplicial complexes and the technology of their ad-theories to construct $L$-theory spectra with good multiplicative properties. As a byproduct they constructed a cohomology theory on the category of ball complexes

$$
X \mapsto H^{n}\left(X ; \mathbf{L}_{\bullet}\right) \cong L_{n}\left(\Lambda^{*}(X)\right),
$$

where $\Lambda^{*}(X)$ is a certain algebraic bordism category (different from $\Lambda_{*}(X)$ ), see Theorem 16.1 and Remark 16.2 in [LM14]. Moreover, in the preprint [BLM14] Banagl, Laures and McClure use the ad-theories to construct a homology theory

$$
Z \mapsto H_{n}\left(Z ; \mathbf{L}^{\bullet}\right)
$$

on the category of topological spaces with the coefficients in the symmetric $L$-theory spectrum $\mathbf{L}^{\bullet}$ and prove a product formula for symmetric signatures of IP-spaces. However, they do not construct a homology theory with the coefficients in the quadratic spectrum L. and they also do not consider the quadratic signature over $X$ map (1.1). Our first aim in this paper is to construct such a homology theory and to construct products:

$$
\begin{aligned}
& -\otimes-: H_{k}\left(L ; \mathbf{L}^{\bullet}\right) \otimes H_{n}\left(K ; \mathbf{L}^{\bullet}\right) \rightarrow H_{n+k}\left(L \times K ; \mathbf{L}^{\bullet}\right), \\
& -\otimes-: H_{k}\left(L ; \mathbf{L}^{\bullet}\right) \otimes H_{n}(K ; \mathbf{L} \bullet) \rightarrow H_{n+k}\left(L \times K ; \mathbf{L}_{\bullet}\right) .
\end{aligned}
$$

Moreover, generalising [Ran92] and [KMM13], given a $k$-manifold $F$ with a map $r_{F}: F \rightarrow L$ to a ball complex $L$ we construct its symmetric signature over $L$ as $\operatorname{ssign}_{L}(F) \in H_{k}\left(L ; \mathbf{L}^{\bullet}\right)$ and given a degree one normal map $(f, b): M \rightarrow X$ between 
$n$-manifolds with a map $r_{X}: X \rightarrow K$ to a ball complex we construct its quadratic signature over $K$ as qsign $K(f, b) \in H_{n}\left(K ; \mathbf{L}_{\bullet}\right)$ and we prove the product formulae:

Theorem 1.1. Let $r_{F}: F \rightarrow L$ be a map from a $k$-dimensional manifold to a ball complex $L$, let $r_{F^{\prime}}: F^{\prime} \rightarrow K$ be a map from an $n$-dimensional manifold to a ball complex $K$ and let $(f, b): M \rightarrow X$ be a degree one normal map between $n$-dimensional manifolds with a map $r_{X}: X \rightarrow K$. The products (1.2) satisfy

$$
\begin{aligned}
\operatorname{ssign}_{L \times K}\left(F \times F^{\prime}\right) & =\operatorname{ssign}_{L}(F) \otimes \operatorname{ssign}_{K}\left(F^{\prime}\right), \\
\operatorname{qsign}_{L \times K}\left(\operatorname{id}_{F} \times f, \operatorname{id}_{\nu_{F}} \times b\right) & =\operatorname{ssign}_{L}(F) \otimes \operatorname{qsign}_{K}(f, b) .
\end{aligned}
$$

The first formula is also proved in [BLM14, Theorem 11.1] for IP-spaces. A special case of the second formula gives that for a closed $k$-dimensional manifold $F$ and a closed $n$-dimensional manifold $X$ we have a commutative diagram

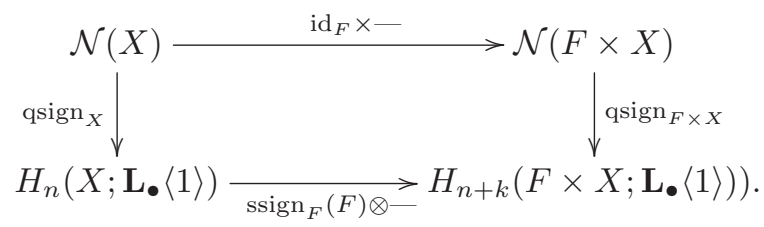

There exist also relative versions of these signatures when $F$ or $X$ have a boundary. Applying the above diagram in the case $(F, \partial F)=\left(D^{k}, S^{k-1}\right)$ yields the suspension isomorphism in the bottom row and hence we obtain a geometric description of such a suspension. If needed, see [Ran92, Chapter 15] for the connective spectra $\mathbf{L} \bullet\langle 1\rangle$.

As noted above, the formulae we obtain are related to products mentioned in [LR92, Appendix], [Ran92, Chapter 21]. In particular, Proposition 21.1 in [Ran92, Chapter 21] states multiplicativity for the visible symmetric signature of a Poincaré complex. The visible symmetric signature is important since it is used to define the total surgery obstruction. Hence it would shed some light on the multiplicative properties of the total surgery obstruction itself. In principle it should be possible to give an easy proof of the formula from Proposition 21.1 in [Ran92, Chapter 21] in our setup, but for such a proof we would also need to formulate the whole algebraic surgery exact sequence for $X$ a ball complex and prove its main properties, which we hope to work out in a future work. Nevertheless, we hope that already the formulae obtained here will have a direct application in a future work along the lines of [KMM13, Section 15] where we aim at simplifying the proof of the main theorem about the total surgery obstruction from [Ran79, KMM13], since the proof uses products on homology and cohomology.

The present paper differs from [LM14, BLM14] in that although we work with ball complexes we do not use the ad-theories. We work instead in the setup of additive categories with chain duality from [Ran92] and [Wei92]. One benefit is that our definitions are generalisations of those from [Ran92] and [Wei92] and so the reader does not have to be familiar with the technology of ad-theories.

The present work is structured as follows. In Section 2 we review facts about ball complexes, mainly using [McC75]. In Sections 3, 4, and 5 we define additive categories $\mathbb{A}_{*}(X)$ and $\mathbb{A}^{*}(X)$ for a ball complex and we show that they possess chain duality in the sense of [Ran92, Chapter 1]. We find both the proof in [Ran92, Chapter 5] and in [Wei92] of this fact in the case $X$ is a simplicial complex or a 
finite $\Delta$-set very dense and hence we give all the details and put the chain duality in a more general context. Besides the product formulae this is meant to be another contribution of our paper. Once the chain duality is set up, we observe in Section 6 that the proof from [Wei92] that the assignment $X \mapsto L_{n}\left(\Lambda_{*}(X)\right)$ defines a homology theory essentially works for ball complexes as well. Finally, in Sections 7 and 8 we prove the desired product formulae for $L$-homology.

\section{Acknowledgments}

The first author would like to thank the University of Glasgow where most of his work for the present article was done. He would also like to thank the Max Planck Institute for Mathematics in Bonn where the first part of this work was done. The second author would like to thank the University of Bonn where this work was started. Later during this work he was supported through the project "Topology of highdimensional manifolds" under the scheme "Returns" of the "Ministry of education, science, research and sport of the Slovak republic", by the grant VEGA 1/0101/17, and by the Slovak Research and Development Agency under the contract No. APVV16-0053.

\section{Ball complexes and related concepts}

We start with a definition and collect some properties of ball complexes and related concepts. The main sources are [McC75, BRS76, LM14].

Definition 2.1. [BRS76, LM14] Let $n$ be a natural number, let $K$ be a finite collection of PL balls in $\mathbb{R}^{n}$ and write $|K|$ for the union $\bigcup_{\sigma \in K} \sigma$. We say that $K$ is a ball complex if the interiors of the balls of $K$ are disjoint and the boundary of each ball of $K$ is a union of balls of $K$.

A ball complex is a regular cell complex in the sense of [Whi78, Section II.6]. To distinguish these from cell complexes in the next definition we will refer to them as regular $C W$-complexes. In order to have good dual cell decompositions we need a refinement. Recall that a polyhedron $X$ is a topological space with a maximal family of triangulations [McC75]. For example, the geometric realisation $|K|$ of a simplicial complex $K$ gives such a polyhedron, so that $K$ is one of the triangulations in the family. A cone on a topological space $X$ is $\operatorname{cone}(X):=X \times[0,1] / \sim$ where $(x, 1) \sim(y, 1)$ for all $x, y \in X$ with $c:=[(x, 1)]$ the cone point.

Definition 2.2. [McC75] A cone complex $\mathcal{C}$ on a polyhedron $X$ is a locally finite covering of $X$ by compact subpolyhedra, together with a subpolyhedron $\partial \alpha$ for each $\alpha \in \mathcal{C}$, such that

1. for each $\alpha \in \mathcal{C}$ we have that $\partial \alpha$ is a union of elements in $\mathcal{C}$

2. for $\alpha \neq \beta$ the interiors $\stackrel{\circ}{\alpha}=(\alpha \backslash \partial \alpha)$ and $\stackrel{\circ}{\beta}=(\beta \backslash \partial \beta)$ satisfy $\stackrel{\circ}{\alpha} \stackrel{\circ}{\beta}=\emptyset$

3. for each $\alpha \in \mathcal{C}$ there is a PL-homeomorphism $\alpha \cong \operatorname{cone}(\partial \alpha)$ rel $\partial \alpha$.

A cell complex is a cone complex such that each cone $\alpha \in \mathcal{C}$ is a ball with $\partial \alpha$ its boundary sphere. A structure for a cone $\alpha \in \mathcal{C}$ is a choice of a homeomorphism 
$f_{\alpha}: \alpha \rightarrow \operatorname{cone}(\partial \alpha)$. The cone point of $\alpha$ in this structure is the point $f_{\alpha}^{-1}(c)$. A structured cone complex is a cone complex with a choice of structure for each cone. A structured cell complex is a cell complex which is structured as a cone complex.

Dual cell decompositions for ball complexes are described in [McC75, p. 274]. They are defined by essentially the same procedure as for simplicial complexes. Our principal motivation for working with structured cell (or ball) complexes rather than simplicial complexes is that structured cell (or ball) complexes behave better with respect to products.

Let $X$ be a ball complex. For each ball $\sigma \in X$ choose a point in its interior. Alternatively assume that $X$ is a structured cell complex, then a choice of such a point is given by taking the cone point for each ball. We obtain the canonical derived subdivision $X^{\prime}$ which is a simplicial complex with $l$-simplices given by sequences $\sigma_{0}<\cdots<\sigma_{l}$ where $\sigma_{i} \in X$, see [McC75, Proposition 2.1]. The dual cell $D(\sigma, X)$ is a subcomplex of $X^{\prime}$ which consists of the simplices in $X^{\prime}$ such that $\sigma \leqslant \sigma_{0}$. The above construction depends on choices of points if $X$ is just a ball complex, or if $X$ is a structured cell complex there are no choices involved, which is from our point of view the main advantage of structured cell complexes. We also note that the space of these choices is contractible which means that from the homotopy theory point of view there is very little difference between the respective categories.

Let $X$ and $F$ be structured cell complexes and let $\sigma \in X$ be an $n$-ball and let $\tau \in F$ be a $k$-ball. Then we have an $(n+k)$-ball $\sigma \times \tau \in X \times F$ and this gives a structured cell complex structure on $X \times F$. In addition we see from the definitions for dual cells that (see also [McC75, Proposition 2.3])

$$
D(\sigma \times \tau, X \times F)=D(\sigma, X) \times D(\tau, F) .
$$

We denote the category of structured cell complexes and inclusions respecting the structurings by $\mathcal{S T} \mathcal{R C} \mathcal{E} \mathcal{L} \mathcal{L}$, the category of ball complexes and inclusions respecting the ball structures by $\mathcal{B} \mathcal{A L} \mathcal{L}$, the category of regular $C W$-complexes and inclusions respecting the cell structures by $\mathcal{R E} \mathcal{G C W}$ (these are called regular cell complexes in [Whi78, Section II.6]) so that we have forgetful functors

$$
\mathcal{S T} \mathcal{R C} \mathcal{E} \mathcal{L} \rightarrow \mathcal{B} \mathcal{A L} \mathcal{L} \rightarrow \mathcal{R E} \mathcal{G C W} .
$$

We finish with a definition and a proposition which we will need later.

Definition 2.3. Let $X$ be a ball complex.

1. Let $\operatorname{st}(\sigma)$ denote the open star of a ball $\sigma$, defined as

$$
\operatorname{st}(\sigma)=\bigcup_{\sigma \leqslant \tau} \stackrel{\circ}{\tau}
$$

2. For all inclusions of balls $\rho \leqslant \sigma$, let $[\rho: \sigma]$ denote the union of interiors of all balls containing $\rho$ and contained in $\sigma$ :

$$
[\rho: \sigma]=\bigcup_{\rho \leqslant \tau \leqslant \sigma} \stackrel{\circ}{\tau}=\operatorname{st}(\rho) \cap \sigma .
$$

Proposition 2.4. For all strict inclusions of balls $\rho<\sigma$ in $X$ the cellular chain complex $C_{*}([\rho: \sigma] ; \mathbb{Z})$ and cochain complex $C^{-*}([\rho: \sigma] ; \mathbb{Z})$ are chain contractible. 
Proof. By definition $[\rho: \sigma]=\operatorname{st}(\rho) \cap \sigma$. When $\rho<\sigma$ in a regular cell complex we have that $\partial \sigma \backslash \operatorname{st}(\rho)$ is a closed $(|\sigma|-1)$-ball. Hence

$$
C_{*}([\rho: \sigma] ; \mathbb{Z})=C_{*}(\sigma, \partial \sigma \backslash \operatorname{st}(\rho) ; \mathbb{Z}) \cong C_{*}\left(D^{|\sigma|}, D^{|\sigma|-1} ; \mathbb{Z}\right) \simeq 0 .
$$

Similarly for $C^{-*}([\rho: \sigma] ; \mathbb{Z})$.

\section{Chain duality}

In defining $L$-theory of a ring $R$ an involution is required. The involution allows one to convert right $R$-modules into left $R$-modules and hence tensor together two right $R$-modules. The $L$-theory of $R$ depends on the choice of involution. When generalising to $L$-theory of an additive category $\mathbb{A}$ one could also use an involution but instead a weaker structure, that of a chain duality on $\mathbb{A}$, is used instead. A chain duality on $\mathbb{A}$ determines an involution on the derived category of chain complexes in $\mathbb{A}$ and chain homotopy classes of chain maps, allowing for the definition of an $n$-dimensional algebraic Poincaré complex in $\mathbb{A}$ as a finite chain complex which is chain equivalent to its $n$-dual. This weakening is crucial for local duality in later sections.

In this section we follow [Ran92, §1] adding some details and explanations. Throughout the paper $\mathbb{A}$ denotes an additive category and $\mathbb{B}(\mathbb{A})$ denotes the additive category of bounded chain complexes in $\mathbb{A}$ together with chain maps. Let $\iota_{\mathbb{A}}: \mathbb{A} \rightarrow$ $\mathbb{B}(\mathbb{A})$ denote inclusion into degree 0 and let $\mathcal{S}_{\mathcal{C}}: \mathcal{C} \times \mathcal{C} \rightarrow \mathcal{C} \times \mathcal{C}$ denote the functor that switches the two factors for any category $\mathcal{C}$. Let $S^{n}: \mathbb{B}(\mathbb{A}) \rightarrow \mathbb{B}(\mathbb{A})$ and $\Sigma^{n}: \mathbb{B}(\mathbb{A}) \rightarrow \mathbb{B}(\mathbb{A})$ be respectively the unsigned and signed suspension functors as in [Ran92, pp. 25-26].

The total complex of a double chain complex can be used to extend an additive contravariant (resp. covariant) functor $T_{\mathbb{A}}: \mathbb{A} \rightarrow \mathbb{B}(\mathbb{A})$ to an additive contravariant (resp. covariant) functor $T_{\mathbb{B}}: \mathbb{B}(\mathbb{A}) \rightarrow \mathbb{B}(\mathbb{A})$ such that $T_{\mathbb{A}}=T_{\mathbb{B}} \circ \iota_{\mathbb{A}}$. The contravariant case is explained in detail in [Ran92, p. 26] and the covariant case is obtained by replacing $C_{-p}$ with $C_{p}$ in all the formulae for the contravariant case.

Proposition 3.1. A natural transformation $e_{\mathbb{A}}: \mathcal{F}_{\mathbb{A}} \Rightarrow \mathcal{G}_{\mathbb{A}}: \mathbb{A} \rightarrow \mathbb{B}(\mathbb{A})$ of additive contravariant (resp. covariant) functors extends to a natural transformation of their extensions $e_{\mathbb{B}}: \mathcal{F}_{\mathbb{B}} \Rightarrow \mathcal{G}_{\mathbb{B}}: \mathbb{B}(\mathbb{A}) \rightarrow \mathbb{B}(\mathbb{A})$.

Proof. For additive contravariant functors setting

$$
e_{\mathbb{B}}(C)_{n}=\sum_{p+q=n} e_{\mathbb{A}}\left(C_{-p}\right)_{q}: \sum_{p+q=n} \mathcal{F}_{\mathbb{A}}\left(C_{-p}\right)_{q} \rightarrow \sum_{p+q=n} \mathcal{G}_{\mathbb{A}}\left(C_{-p}\right)_{q}
$$

defines the required natural transformation and for additive covariant functors replace all instances of $C_{-p}$ with $C_{p}$ in the above.

Definition 3.2. A chain duality on an additive category $\mathbb{A}$ is a pair $\left(T_{\mathbb{A}}, e_{\mathbb{A}}\right)$ where

- $T_{\mathbb{A}}$ is a contravariant additive functor $T_{\mathbb{A}}: \mathbb{A} \rightarrow \mathbb{B}(\mathbb{A})$,

- $e_{\mathbb{A}}$ is a natural transformation $e_{\mathbb{A}}: T_{\mathbb{B}} \circ T_{\mathbb{A}} \Rightarrow \iota_{\mathbb{A}}$ such that, for all $M \in \mathbb{A}$ :

$-e_{\mathbb{B}}\left(T_{\mathbb{A}}(M)\right) \circ T_{\mathbb{B}}\left(e_{\mathbb{A}}(M)\right)=\operatorname{id}_{T_{\mathbb{A}}(M)}: T_{\mathbb{A}}(M) \rightarrow T_{\mathbb{B}}^{2}\left(T_{\mathbb{A}}(M)\right) \rightarrow T_{\mathbb{A}}(M)$,

$-e_{\mathbb{A}}(M): T_{\mathbb{B}}\left(T_{\mathbb{A}}(M)\right) \rightarrow M$ is a chain equivalence. 
The dual $T_{\mathbb{A}}(M)$ of an object $M$ is a chain complex in $\mathbb{A}$ and the extension $T_{\mathbb{B}}$ defines the dual of a chain complex $C \in \mathbb{B}(\mathbb{A})$ as $T_{\mathbb{B}}(C)$. A chain duality is used to define a tensor product of two objects $M, N \in \mathbb{A}$ over $\mathbb{A}$ as

$$
M \otimes_{\mathbb{A}} N=\operatorname{Hom}_{\mathbb{A}}\left(T_{\mathbb{A}}(M), N\right),
$$

which is a priori just a chain complex in the category $\mathrm{Ab}$ of Abelian groups. This generalises to chain complexes $C, D \in \mathbb{B}(\mathbb{A})$ by setting

$$
C \otimes_{\mathbb{A}} D=\operatorname{Hom}_{\mathbb{A}}\left(T_{\mathbb{B}}(C), D\right) .
$$

See $\left[\right.$ Ran92, p. 26] for the definition of $\operatorname{Hom}_{\mathbb{A}}(C, D)$ as the total complex of a double complex.

The tensor product $f \otimes_{\mathbb{A}} f^{\prime}: C \otimes_{\mathbb{A}} C^{\prime} \rightarrow D \otimes_{\mathbb{A}} D^{\prime}$ of a pair of morphisms $f: C \rightarrow$ $D, f^{\prime}: C^{\prime} \rightarrow D^{\prime}$ in $\mathbb{B}(\mathbb{A})$ sends $\phi \in \operatorname{Hom}_{\mathbb{A}}\left(T_{\mathbb{B}}(C), C^{\prime}\right)$ to

$$
\left(f \otimes_{\mathbb{A}} f^{\prime}\right)(\phi):=T_{\mathbb{B}}(f)^{*}\left(f^{\prime}\right)_{*}(\phi)=f^{\prime} \circ \phi \circ T_{\mathbb{B}}(f) \in \operatorname{Hom}_{\mathbb{A}}\left(T_{\mathbb{B}}\left(C^{\prime}\right), D^{\prime}\right) .
$$

Tensor product over $\mathbb{A}$ thus defines a functor

$$
-\otimes_{\mathbb{A}}-: \mathbb{B}(\mathbb{A}) \times \mathbb{B}(\mathbb{A}) \rightarrow \mathbb{B}(\mathrm{Ab}) .
$$

Example 3.3. For a ring $R$ let $\mathbb{A}(R)$ denote the additive category of f.g. free left $R$ modules. Let $T=\operatorname{Hom}_{R}(-, R): \mathbb{A}(R) \rightarrow \mathbb{A}(R)$. Then there is a natural isomorphism $e: T^{2} \Rightarrow \operatorname{id}_{\mathbb{A}(R)}$ with $e(M)=\operatorname{ev}(M)^{-1}$ where

$$
\begin{aligned}
\operatorname{ev}(M): M & \stackrel{\cong}{\mapsto} T^{2}(M)=\operatorname{Hom}_{R}\left(\operatorname{Hom}_{R}(M, R), R\right), \\
m & \mapsto(f \mapsto f(m)) .
\end{aligned}
$$

One can easily check that $e(T(M)) \circ T(e(M))=\operatorname{id}_{T(M)}$ for all $M \in \mathbb{A}(R)$ and thus that $(T, e)$ is a chain duality on $\mathbb{A}(R)$.

There is also a tensor product of f.g. free left $R$-modules such that

$$
M \otimes_{R} M^{\prime} \cong \operatorname{Hom}_{R}\left(T(M), M^{\prime}\right),
$$

with switch isomorphisms $T_{M, M^{\prime}}: M \otimes_{R} M^{\prime} \stackrel{\cong}{\longrightarrow} M^{\prime} \otimes_{R} M$ corresponding to isomorphisms

$$
\begin{aligned}
\operatorname{Hom}_{R}\left(T(M), M^{\prime}\right) & \cong \operatorname{Hom}_{R}\left(T\left(M^{\prime}\right), M\right), \\
\phi & \mapsto e(M) \circ T(\phi) .
\end{aligned}
$$

The previous example is a special case of a chain duality, in the sense that duals are only modules rather than chain complexes. It does, however, motivate how a chain duality $\left(T_{\mathbb{A}}, e_{\mathbb{A}}\right)$ on an additive category $\mathbb{A}$ is used to define a tensor product by (3.1) together with switch isomorphisms

$$
T_{C, D}: C \otimes_{\mathbb{A}} D \stackrel{\cong}{\longrightarrow} D \otimes_{\mathbb{A}} C,
$$

such that $T_{D, C}=T_{C, D}^{-1}$, for all $C, D \in \mathbb{B}(\mathbb{A})$. The idea is to use $T_{\mathbb{A}}$ and $e_{\mathbb{A}}$ as in (3.2). This is explained in the following propositions.

Proposition 3.4. Let $T_{\mathbb{A}}: \mathbb{A} \rightarrow \mathbb{B}(\mathbb{A})$ be a contravariant additive functor with extension $T_{\mathbb{B}}$. Then, for all $C, D \in \mathbb{B}(\mathbb{A})$, the extension $T_{\mathbb{B}}$ induces a chain map

$$
T_{\mathbb{B}}: \operatorname{Hom}_{\mathbb{A}}(C, D) \rightarrow \operatorname{Hom}_{\mathbb{A}}\left(T_{\mathbb{B}}(D), T_{\mathbb{B}}(C)\right) .
$$


Proof. The chain map is defined by specifying the components $T_{\mathbb{B}}(\phi)_{p, q}^{r, s}: T_{\mathbb{A}}\left(D_{p}\right)_{q} \rightarrow$ $T_{\mathbb{A}}\left(C_{r}\right)_{s}$ as

$$
\left(T_{\mathbb{B}}\right)_{n}(\phi)_{p, q}^{r, s}= \begin{cases}(-1)^{n q}(-1)^{\frac{1}{2} n(n-1)} T_{\mathbb{A}}\left(\phi_{p-n}^{p}\right)_{q}, & s=q, r=p-n \\ 0, & \text { otherwise }\end{cases}
$$

Remark 3.5. Let $\phi: C \rightarrow D$ be a morphism of $\mathbb{B}(\mathbb{A})$, i.e. a 0 -cycle $\phi \in \operatorname{Hom}_{\mathbb{A}}(C, D)_{0}$. Then $\left(T_{\mathbb{B}}\right)_{0}(\phi) \in \operatorname{Hom}_{\mathbb{A}}\left(T_{\mathbb{B}}(D), T_{\mathbb{B}}(C)\right)_{0}$ is precisely the image $T_{\mathbb{B}}(\phi)$ of $\phi$ under the extended functor $T_{\mathbb{B}}: \mathbb{B}(\mathbb{A}) \rightarrow \mathbb{B}(\mathbb{A})$.

Proposition 3.6. Let $\phi \in \operatorname{Hom}_{\mathbb{A}}(C, D)_{0}$ be a chain equivalence. Then $\left(T_{\mathbb{B}}\right)_{0}(\phi) \in$ $\operatorname{Hom}_{\mathbb{A}}\left(T_{\mathbb{B}}(D), T_{\mathbb{B}}(C)\right)_{0}$ is also a chain equivalence.

Proof. Applying $\left(T_{\mathbb{B}}\right)_{0}$ to the chain map $\phi$ and its inverse and applying $\left(T_{\mathbb{B}}\right)_{1}$ to the chain homotopies gives the chain map $\left(T_{\mathbb{B}}\right)_{0}(\phi)$, its inverse and the corresponding chain homotopies.

Proposition 3.7. A contravariant additive functor $T_{\mathbb{A}}: \mathbb{A} \rightarrow \mathbb{B}(\mathbb{A})$ with extension $T_{\mathbb{B}}$ induces a natural transformation also denoted $T_{\mathbb{B}}$ :

$$
T_{\mathbb{B}}:-\otimes_{\mathbb{A}}-\Rightarrow\left(-\otimes_{\mathbb{A}}-\right) \circ\left(\operatorname{id}_{\mathbb{B}(\mathbb{A})} \times T_{\mathbb{B}}^{2}\right) \circ \mathcal{S}_{\mathbb{B}(\mathbb{A})}: \mathbb{B}(\mathbb{A}) \times \mathbb{B}(\mathbb{A}) \rightarrow \mathbb{B}(\mathrm{Ab})
$$

by

$$
T_{\mathbb{B}}(C, D)=T_{\mathbb{B}}: C \otimes_{\mathbb{A}} D \rightarrow D \otimes T_{\mathbb{B}}^{2}(C),
$$

where $T_{\mathbb{B}}$ is the chain map of Proposition 3.4 with $T_{\mathbb{B}}(C)$ in place of $C$.

Proof. This is a straightforward verification using the formulae for $f^{\prime} \otimes_{\mathbb{A}} T_{\mathbb{B}}^{2}(f)$ and $f \otimes_{\mathbb{A}} f^{\prime}$ together with (3.3).

Proposition 3.8. For all $C, D \in \mathbb{B}(\mathbb{A})$, let $T_{C, D}: C \otimes_{\mathbb{A}} D \rightarrow D \otimes_{\mathbb{A}} C$ be defined as the composition

$$
T_{C, D}=\left(\operatorname{id}_{D} \otimes_{\mathbb{A}} e_{\mathbb{B}}(C)\right) \circ T_{\mathbb{B}}: C \otimes_{\mathbb{A}} D \rightarrow D \otimes_{\mathbb{A}} T_{\mathbb{B}}^{2}(C) \rightarrow D \otimes_{\mathbb{A}} C,
$$

where $T_{\mathbb{B}}: C \otimes_{\mathbb{A}} D \rightarrow D \otimes_{\mathbb{A}} T_{\mathbb{B}}^{2}(C)$ is the chain map of Proposition 3.4 with $T_{\mathbb{B}}(C)$ in place of $C$. Then $T_{C, D}$ is a chain isomorphism with inverse $T_{D, C}$.

Proof. $T_{C, D}$ is the composition of two chain maps and hence a chain map. The fact that $T_{D, C} \circ T_{C, D}=\operatorname{id}_{C \otimes_{\mathbb{A}} D}$ follows from the properties of $\left(T_{\mathbb{A}}, e_{\mathbb{A}}\right)$ being a chain duality on $\mathbb{A}$. This uses naturality of $e_{\mathbb{A}}$ and that for all $p \in \mathbb{Z}$ we have

$$
e_{\mathbb{B}}\left(T_{\mathbb{A}}\left(C_{p}\right)\right) \circ T_{\mathbb{B}}\left(e_{\mathbb{A}}\left(C_{p}\right)\right)=\operatorname{id}_{T_{\mathbb{A}}\left(C_{p}\right)}: T_{\mathbb{A}}\left(C_{p}\right) \rightarrow T_{\mathbb{B}}^{2}\left(T_{\mathbb{A}}\left(C_{p}\right)\right) \rightarrow T_{\mathbb{A}}\left(C_{p}\right) .
$$

Remark 3.9. By Remark 3.5 the identity chain map $\operatorname{id}_{T_{\mathbb{B}}(C)}$ is sent by

$$
T_{\mathbb{B}}: \operatorname{Hom}_{\mathbb{A}}\left(T_{\mathbb{B}}(C), T_{\mathbb{B}}(C)\right) \rightarrow \operatorname{Hom}_{\mathbb{A}}\left(T_{\mathbb{B}}^{2}(C), T_{\mathbb{B}}^{2}(C)\right)
$$

to $T_{\mathbb{B}}\left(\mathrm{id}_{T_{\mathbb{B}}(C)}\right)=\mathrm{id}_{T_{\mathbb{B}}^{2}(C)}$. Consequently, by definition of $T_{C, T_{\mathbb{B}}(C)}$ we have

$$
T_{C, T_{\mathbb{B}}(C)}\left(\mathrm{id}_{T_{\mathbb{B}}(C)}\right)=e_{\mathbb{B}}(C) \circ T_{\mathbb{B}}\left(\mathrm{id}_{T_{\mathbb{B}}(C)}\right)=e_{\mathbb{B}}(C) .
$$

Thus $e_{\mathbb{B}}(C)$ can be recovered from the switch isomorphisms. 
Proposition 3.10. A chain duality on $\mathbb{A}$ defines a natural isomorphism

$$
T_{-,-}:-\otimes_{\mathbb{A}}-\Rightarrow\left(-\otimes_{\mathbb{A}}-\right) \circ \mathcal{S}_{\mathbb{B}(\mathbb{A})}: \mathbb{B}(\mathbb{A}) \times \mathbb{B}(\mathbb{A}) \rightarrow \mathbb{B}(\mathrm{Ab}),
$$

such that

$$
\left(T_{-,-} \circ \operatorname{id}_{\mathcal{S}_{\mathbb{B}(\mathbb{A})}}\right) \circ T_{-,-}:-\otimes_{\mathbb{A}}-\Rightarrow\left(-\otimes_{\mathbb{A}}-\right) \circ \mathcal{S}_{\mathbb{B}(\mathbb{A})}^{2}=\left(-\otimes_{\mathbb{A}}-\right)
$$

is the identity natural isomorphism of the functor $-\otimes_{\mathbb{A}}-$.

Proof. For all $C, D \in \mathbb{B}(\mathbb{A})$, define $T_{-,-}(C, D)=T_{C, D}$ as in Proposition 3.8. The fact that $T_{-,-}$is a natural transformation follows directly from the fact that $e_{\mathbb{B}}$ and the $T_{\mathbb{B}}$ of Proposition 3.7 are natural transformations. The second part follows from the fact that $T_{D, C} \circ T_{C, D}=\operatorname{id}_{C \otimes_{\mathbb{A}} D}$.

We have just seen that a chain duality $\left(T_{\mathbb{A}}, e_{\mathbb{A}}\right)$ is precisely the additional structure on an additive category $\mathbb{A}$ required so that if we define a tensor product over $\mathbb{A}$ by $-\otimes_{\mathbb{A}}-=\operatorname{Hom}_{\mathbb{A}}\left(T_{\mathbb{B}}(-),-\right)$ there is a natural switch isomorphism $T_{-,-}:-\otimes_{\mathbb{A}}-\Rightarrow$ $\left(-\otimes_{\mathbb{A}}-\right) \circ \mathcal{S}_{\mathbb{B}(\mathbb{A})}$ with the property that $T_{D, C}=T_{C, D}^{-1}$ for all $C, D \in \mathbb{B}(\mathbb{A})$.

Conversely, suppose one starts with a contravariant functor $T_{\mathbb{A}}: \mathbb{A} \rightarrow \mathbb{B}(\mathbb{A})$ and a natural switch isomorphism $T_{-,-}:-\otimes_{\mathbb{A}}-\Rightarrow\left(-\otimes_{\mathbb{A}}-\right) \circ \mathcal{S}_{\mathbb{B}(\mathbb{A})}$ with the property that $T_{D, C}=T_{C, D}^{-1}$ for all $C, D \in \mathbb{B}(\mathbb{A})$. Then $T_{\mathbb{A}}$ and the switch isomorphism can be used to define a natural transformation $e_{\mathbb{A}}: T_{\mathbb{B}} \circ T_{\mathbb{A}} \rightarrow \iota_{\mathbb{A}}$ such that $\left(T_{\mathbb{A}}, e_{\mathbb{A}}\right)$ satisfies all the properties of being a chain duality, except possibly that $e_{\mathbb{A}}(M): T_{\mathbb{B}}\left(T_{\mathbb{A}}(M)\right) \rightarrow$ $M$ is a chain equivalence for all $M$.

Proposition 3.11. Let $T_{\mathbb{A}}: \mathbb{A} \rightarrow \mathbb{B}(\mathbb{A})$ be a contravariant additive functor and let

$$
-\otimes_{\mathbb{A}}-=\operatorname{Hom}_{\mathbb{A}}\left(T_{\mathbb{B}}(-),-\right): \mathbb{B}(\mathbb{A}) \times \mathbb{B}(\mathbb{A}) \rightarrow \mathbb{B}(\mathrm{Ab}) .
$$

Suppose there exists a natural isomorphism

$$
T_{-,-}:-\otimes_{\mathbb{A}}-\Rightarrow\left(-\otimes_{\mathbb{A}}-\right) \circ \mathcal{S}_{\mathbb{B}(\mathbb{A})},
$$

such that $T_{D, C} \circ T_{C, D}=\operatorname{id}_{C \otimes_{\mathbb{A}} D}$ for all $C, D \in \mathbb{B}(\mathbb{A})$. Let

$$
e_{\mathbb{B}}(C)=T_{C, T_{\mathbb{B}}(C)}\left(\operatorname{id}_{T_{\mathbb{B}}(C)}\right),
$$

for all $C \in \mathbb{B}(\mathbb{A})$. Then

1. this defines a natural transformation $e_{\mathbb{B}}: T_{\mathbb{B}}^{2} \Rightarrow \mathrm{id}_{\mathbb{B}(\mathbb{A})}$,

2. $T_{C, D}(\phi)=e_{\mathbb{B}}(C) \circ T_{\mathbb{B}}(\phi)$, for all chain maps $\phi: T_{\mathbb{B}}(C) \rightarrow D$,

3. $e_{\mathbb{B}}\left(T_{\mathbb{B}}(C)\right) \circ T_{\mathbb{B}}\left(e_{\mathbb{B}}(C)\right)=\mathrm{id}_{T_{\mathbb{B}}(C)}$, for all $C \in \mathbb{B}(\mathbb{A})$.

Proof. 1. By naturality of $T_{-,-}$we have that

$$
\begin{aligned}
\left(\operatorname{id}_{T_{\mathbb{B}}(C)} \otimes_{\mathbb{A}} f\right) \circ T_{C, T_{\mathbb{B}}(C)} & =T_{D, T_{\mathbb{B}}(C)} \circ\left(f \otimes_{\mathbb{A}} \operatorname{id}_{T_{\mathbb{B}}(C)}\right), \\
\left(T_{\mathbb{B}}(f) \otimes_{\mathbb{A}} \operatorname{id}_{D}\right) \circ T_{D, T_{\mathbb{B}}(D)} & =T_{D, T_{\mathbb{B}}(C)} \circ\left(\operatorname{id}_{D} \otimes_{\mathbb{A}} T_{\mathbb{B}}(f)\right),
\end{aligned}
$$

both hold for all chain maps $f: C \rightarrow D$. Applying these equations respectively to $\operatorname{id}_{T_{\mathbb{B}}(C)} \in C \otimes_{\mathbb{A}} T_{\mathbb{B}}(C)$ and $\operatorname{id}_{T_{\mathbb{B}}(D)} \in D \otimes_{\mathbb{A}} T_{\mathbb{B}}(D)$ proves naturality of $e_{\mathbb{B}}$.

2. By naturality of $T_{-,-}$we have that

$$
\left(\phi \otimes_{\mathbb{A}} \operatorname{id}_{C}\right) \circ T_{C, T_{\mathbb{B}}(C)}=T_{C, D} \circ\left(\operatorname{id}_{C} \otimes_{\mathbb{A}} \phi\right),
$$

for all chain maps $\phi: T_{\mathbb{B}}(C) \rightarrow D$. The Applying this equation to $\operatorname{id}_{T_{\mathbb{B}}(C)} \in$ $C \otimes_{\mathbb{A}} T_{\mathbb{B}}(C)$ gives the result. 
3. As $T_{C, D}: C \otimes_{\mathbb{A}} D \rightarrow D \otimes_{\mathbb{A}} C$ is a chain map for all $C, D \in \mathbb{B}(\mathbb{A})$ it follows that $\left(T_{C, D}\right)_{0}$ sends chain maps to chain maps. Hence $e_{\mathbb{B}}(C)=T_{C, T_{\mathbb{B}}(C)}\left(\operatorname{id}_{C \otimes_{\mathbb{A}} T_{\mathbb{B}}(C)}\right)$ is a chain map. The result then follows from the equality

$$
T_{T_{\mathbb{B}}(C), C}\left(T_{C, T_{\mathbb{B}}(C)}\left(\operatorname{id}_{C \otimes_{\mathbb{A}} T_{\mathbb{B}}(C)}\right)\right)=\operatorname{id}_{C \otimes_{\mathbb{A}} T_{\mathbb{B}}(C)}
$$

and part (2) applied to $\phi=e_{\mathbb{B}}(C)$.

The previous proposition indicates a strategy for defining a chain duality on an additive category $\mathbb{A}$ :

1. Define an additive contravariant functor $T_{\mathbb{A}}: \mathbb{A} \rightarrow \mathbb{B}(\mathbb{A})$ which yields the extension $T_{\mathbb{B}}: \mathbb{B}(\mathbb{A}) \rightarrow \mathbb{B}(\mathbb{A})$.

2. Define the tensor product over $\mathbb{A}$ using $T_{\mathbb{B}}$ by $-\otimes_{\mathbb{A}}-=\operatorname{Hom}_{\mathbb{A}}\left(T_{\mathbb{B}}(-),-\right)$.

3. Exhibit a natural isomorphism $T_{-,-}:-\otimes_{\mathbb{A}}-\Rightarrow\left(-\otimes_{\mathbb{A}}-\right) \circ \mathcal{S}_{\mathbb{B}(\mathbb{A})}$ with the property that $T_{D, C}=T_{C, D}^{-1}$ for all $C, D \in \mathbb{B}(\mathbb{A})$.

4. Define $e_{\mathbb{B}}: T_{\mathbb{B}}^{2} \rightarrow \operatorname{id}_{\mathbb{B}(\mathbb{A})}$ by $e_{\mathbb{B}}(C)=T_{C, T_{\mathbb{B}}(C)}\left(\operatorname{id}_{C \otimes_{\mathbb{A}} T_{\mathbb{B}}(C)}\right)$.

5. Prove that $e_{\mathbb{B}}(C): T_{\mathbb{B}}^{2}(C) \rightarrow C$ is a chain equivalence, for all $C \in \mathbb{B}(\mathbb{A})$.

6. Using Proposition 3.11 the pair $\left(T_{\mathbb{B}}, e_{\mathbb{B}}\right)$ is a chain duality on $\mathbb{B}(\mathbb{A})$ and restricting to $\mathbb{A} \subset \mathbb{B}(\mathbb{A})$ this gives a chain duality $\left(T_{\mathbb{A}}, e_{\mathbb{A}}\right)$ on $\mathbb{A}$.

This strategy is employed in Section 5 to define a chain duality on additive categories over ball complexes.

\section{Categories over ball complexes}

In this section we introduce the categories $\mathbb{A}^{*}[X], \mathbb{A}_{*}[X], \mathbb{A}^{*}(X)$ and $\mathbb{A}_{*}(X)$ and develop some of the tools necessary to define a chain duality on $\mathbb{A}^{*}(X)$ or $\mathbb{A}_{*}(X)$. Much of the content can be found in [Ran92]; any differences are clearly indicated.

Let $X$ be a ball complex and $\mathbb{A}$ an additive category.

Definition 4.1. A ball complex $X$ is regarded as a category with objects the set of balls $\sigma \in X$ and morphisms $\tau \rightarrow \sigma$ for all inclusions $\tau \leqslant \sigma$.

Definition 4.2. [Ran92, Definition 4.3] Let $\mathbb{A}^{*}[X]$ and $\mathbb{A}_{*}[X]$ denote the additive categories whose objects are respectively covariant and contravariant functors $M: X \rightarrow \mathbb{A}$ and whose morphisms are natural transformations of such functors.

Definition 4.3. Let $\mathcal{F}: \mathbb{A} \rightarrow \mathbb{A}^{\prime}$ and $\mathcal{G}: \mathbb{A} \rightarrow \mathbb{A}^{\prime}$ be covariant and contravariant functors respectively. Then post-composition with $\mathcal{F}$ or $\mathcal{G}$ defines the following pushforward functors

$$
\begin{array}{cc}
\mathcal{F}_{*}: \mathbb{A}^{*}[X] \rightarrow\left(\mathbb{A}^{\prime}\right)^{*}[X], & \mathcal{F}_{*}: \mathbb{A}_{*}[X] \rightarrow\left(\mathbb{A}^{\prime}\right)_{*}[X], \\
\mathcal{G}_{*}: \mathbb{A}^{*}[X] \rightarrow\left(\mathbb{A}^{\prime}\right)_{*}[X], & \mathcal{G}_{*}: \mathbb{A}_{*}[X] \rightarrow\left(\mathbb{A}^{\prime}\right)^{*}[X] .
\end{array}
$$

Remark 4.4. A bounded chain complex in $\mathbb{A}_{*}[X]$ is just an object in $\mathbb{B}(\mathbb{A})_{*}[X]$ and similarly for chain maps, so that $\mathbb{B}\left(\mathbb{A}_{*}[X]\right)=\mathbb{B}(\mathbb{A})_{*}[X]$. Similarly we have $\mathbb{B}\left(\mathbb{A}^{*}[X]\right)=$ $\mathbb{B}(\mathbb{A})^{*}[X]$. 


\section{Definition 4.5.}

1. An object $M \in \mathbb{A}$ is $X$-based if it is expressed as a direct sum

$$
M=\sum_{\sigma \in X} M(\sigma)
$$

of objects $M(\sigma) \in \mathbb{A}$. A morphism $f: M \rightarrow N$ of $X$-based objects is a collection of morphisms in $\mathbb{A}$

$$
f=\left\{f_{\tau, \sigma}: M(\sigma) \rightarrow N(\tau): \sigma, \tau \in X\right\} .
$$

2. Let the $X$-graded category $\mathbb{G}_{X}(\mathbb{A})$ be the additive category of $X$-based objects of $\mathbb{A}$ and morphisms $f: M \rightarrow N$ of $X$-based objects of $\mathbb{A}$.

The composition of morphisms $f: L \rightarrow M, g: M \rightarrow N$ in $\mathbb{G}_{X}(\mathbb{A})$ is the morphism $g \circ f: L \rightarrow N$ defined by

$$
(g \circ f)_{\rho, \sigma}=\sum_{\tau \in X} g_{\rho, \tau} f_{\tau, \sigma}: L(\sigma) \rightarrow N(\rho) .
$$

3. Let $\left\{\begin{array}{l}\mathbb{A}^{*}(X) \\ \mathbb{A}_{*}(X)\end{array}\right.$ denote the additive category of $X$-based objects $M$ in $\mathbb{A}$ with morphisms $f: M \rightarrow N$ such that $f_{\tau, \sigma}: M(\sigma) \rightarrow N(\tau)$ is zero unless $\left\{\begin{array}{l}\tau \leqslant \sigma \\ \tau \geqslant \sigma .\end{array}\right.$

Notation 4.6. Let $\mathbb{A}(X)$ denote either $\mathbb{A}^{*}(X)$ or $\mathbb{A}_{*}(X)$ and $\mathbb{A}[X]$ either $\mathbb{A}^{*}[X]$ or $\mathbb{A}_{*}[X]$ when it doesn't matter which category is used. When this shorthand is used multiple times in a statement it is assumed that the same choice of upper or lower star is consistently made.

Definition 4.7. Let $X$ be a ball complex and let $Z \subseteq Y \subseteq X$ so that $(Y, Z)$ is a ball complex pair. Define the restriction to $(Y, Z)$

$$
-l_{(Y, Z)}: \mathbb{A}(X) \rightarrow \mathbb{A}(Y) \subset \mathbb{A}(X)
$$

by

$$
\begin{aligned}
\left.M\right|_{(Y, Z)}(\sigma) & =M(\sigma), \quad \forall \sigma \in Y, \sigma \notin Z, \\
\left.M\right|_{(Y, Z)}(f)_{\tau, \sigma} & =f_{\tau, \sigma}, \quad \forall \tau, \sigma \in Y, \tau, \sigma \notin Z .
\end{aligned}
$$

Denote the restriction to $(Y, \partial Y)$ by $-\left.\right|_{Y}$ and the restriction to $(Y, \emptyset)$ by $-\left.\right|_{Y}$.

Definition 4.8. Define the total assembly functor Ass: $\mathbb{G}_{X}(\mathbb{A}) \rightarrow \mathbb{A}$ by

$$
\begin{aligned}
\operatorname{Ass}(M) & =\sum_{\sigma \in X} M(\sigma), \\
\operatorname{Ass}(f: M \rightarrow N) & =\left\{f_{\tau, \sigma}\right\}_{\tau, \sigma \in X}: \sum_{\sigma \in X} M(\sigma) \rightarrow \sum_{\tau \in X} N(\tau) .
\end{aligned}
$$

Note that total assembly is an equivalence of additive categories. Also denote by Ass the total assembly restricted to $\mathbb{A}^{*}(X)$ and $\mathbb{A}_{*}(X)$.

Remark 4.9. Let $\mathbb{M}(\mathbb{Z})$ denote the additive category of left $\mathbb{Z}$-modules. Following [Ran92] we write

$$
\left\{\begin{array} { l } 
{ \mathbb { Z } ^ { * } ( X ) = \mathbb { M } ( \mathbb { Z } ) ^ { * } ( X ) , } \\
{ \mathbb { Z } _ { * } ( X ) = \mathbb { M } ( \mathbb { Z } ) _ { * } ( X ) , }
\end{array} \quad \left\{\begin{array}{l}
\mathbb{Z}^{*}[X]=\mathbb{M}(\mathbb{Z})^{*}[X] \\
\mathbb{Z}_{*}[X]=\mathbb{M}(\mathbb{Z})_{*}[X]
\end{array}\right.\right.
$$


Example 4.10. Let $X$ be a ball complex and let $\mathbb{A}(\mathbb{Z})$ be as defined in Example 3.3 for the ring of integers $\mathbb{Z}$.

The cellular chain complex $C_{*}(X ; \mathbb{Z})$ of $X$ is naturally a finite chain complex in $\mathbb{A}(\mathbb{Z})^{*}(X)$ with $C_{*}(X ; \mathbb{Z})(\sigma)=S^{|\sigma|} \mathbb{Z}$, for all $\sigma \in X$.

The cellular cochain complex $C^{-*}(X ; \mathbb{Z})$ of $X$ is naturally a finite chain complex in $\mathbb{A}(\mathbb{Z})_{*}(X)$ with $C^{-*}(X ; \mathbb{Z})(\sigma)=S^{-|\sigma|} \mathbb{Z}$, for all $\sigma \in X$.

Example 4.11. Let $X^{\prime}$ denote the canonical derived subdivision of a structured ball complex $X$. Then the cellular chain and cochain complexes of $X^{\prime}$ can be viewed as chain complexes in $\mathbb{B}\left(\mathbb{A}(\mathbb{Z})_{*}(X)\right)$ and $\mathbb{B}\left(\mathbb{A}(\mathbb{Z})^{*}(X)\right)$ respectively as follows.

Let $D \in \mathbb{B}\left(\mathbb{A}(\mathbb{Z})_{*}(X)\right)$ be the chain complex with

$$
D(\sigma)=C_{*}(D(\sigma, X), \partial D(\sigma, X) ; \mathbb{Z})
$$

and differential $\left(d_{D}\right)_{\tau, \sigma}$ obtained by assembling $d_{C_{*}\left(X^{\prime}\right)}$ appropriately. Then $\operatorname{Ass}(D)=$ $C_{*}\left(X^{\prime}\right)$.

Similarly the chain complex $D^{*} \in \mathbb{B}\left(\mathbb{A}(\mathbb{Z})^{*}(X)\right)$ with

$$
D^{*}(\sigma)=C^{-*}(D(\sigma, X), \partial D(\sigma, X) ; \mathbb{Z})
$$

and differential $\left(d_{D^{*}}\right)_{\tau, \sigma}$ obtained by assembling $d_{C^{-*}\left(X^{\prime}\right)}$ appropriately is such that $\operatorname{Ass}\left(D^{*}\right)=C^{-*}\left(X^{\prime}\right)$.

Example 4.11 will be generalised in Section 7 to a manifold $F$ equipped with a reference map $r_{F}: F \rightarrow L$ to a ball complex. The preimages of dual cells in $L$ are used to dissect $F$.

Definition 4.12. Let $C(X)$ denote either the $\mathbb{Z}$-coefficient cellular chain complex $C_{*}(X ; \mathbb{Z}) \in \mathbb{B}\left(\mathbb{A}(\mathbb{Z})^{*}(X)\right)$ or cochain complex $C^{-*}(X ; \mathbb{Z}) \in \mathbb{B}\left(\mathbb{A}(\mathbb{Z})_{*}(X)\right)$.

The following proposition illustrates a very important property of the categories $\mathbb{A}^{*}(X)$ and $\mathbb{A}_{*}(X)$. It can be thought of as being analogous to the statement in linear algebra that a triangular matrix is invertible if and only if all its diagonal entries are.

\section{Proposition 4.13.}

1. A chain map $f: C \rightarrow D$ in $\mathbb{A}(X)$ is a chain isomorphism if and only if $f_{\sigma, \sigma}: C(\sigma) \rightarrow D(\sigma)$ is a chain isomorphism in $\mathbb{A}$ for all $\sigma \in X$.

2. A chain complex $C$ in $\mathbb{A}(X)$ is chain contractible if and only if $C(\sigma)$ is chain contractible in $\mathbb{A}$ for all $\sigma \in X$.

3. A chain map $f: C \rightarrow D$ of chain complexes in $\mathbb{A}(X)$ is a chain equivalence if and only if $f_{\sigma, \sigma}: C(\sigma) \rightarrow D(\sigma)$ is a chain equivalence in $\mathbb{A}$ for all $\sigma \in X$.

Proof. These results are well-known for $X$ a simplicial complex (see for example Proposition 4.7 of [Ran92] for parts (2) and (3)); Proposition 7.26 of [AF12] contains parts (2) and (3), the proof contains the correct formulae but falsely asserts in the last line of page 68 that away from the diagonal $d P=P d=0$, the correct statement is that $d P+P d=0$. The simplicial complex proof generalises verbatim to ball complexes. 
Definition 4.14. Define covariant functors $\mathcal{I}_{X, \mathbb{A}}: \mathbb{A}(X) \rightarrow \mathbb{A}[X]$ by sending an object $M \in \mathbb{A}(X)$ to the functor $\mathcal{I}_{X, \mathbb{A}}(M): X \rightarrow \mathbb{A}$ which sends $\sigma \in X$ to

$$
\mathcal{I}_{X, \mathbb{A}}(M)(\sigma)= \begin{cases}\sum_{\rho \leqslant \sigma} M(\rho), & \mathbb{A}(X)=\mathbb{A}^{*}(X), \\ \sum_{\sigma \leqslant \rho} M(\rho), & \mathbb{A}(X)=\mathbb{A}_{*}(X)\end{cases}
$$

and a morphism $\tau \rightarrow \sigma$ in $X$ to the inclusion map

$$
\mathcal{I}_{X, \mathbb{A}}(M)(\tau \rightarrow \sigma): \begin{cases}\mathcal{I}_{X, \mathbb{A}}(M)(\tau) \hookrightarrow \mathcal{I}_{X, \mathbb{A}}(M)(\sigma), & \mathbb{A}(X)=\mathbb{A}^{*}(X), \\ \mathcal{I}_{X, \mathbb{A}}(M)(\sigma) \hookrightarrow \mathcal{I}_{X, \mathbb{A}}(M)(\tau), & \mathbb{A}(X)=\mathbb{A}_{*}(X) .\end{cases}
$$

A morphism $f: M \rightarrow N$ in $\mathbb{A}(X)$ is sent to the natural transformation

$$
\mathcal{I}_{X, \mathbb{A}}(f): \mathcal{I}_{X, \mathbb{A}}(M) \Rightarrow \mathcal{I}_{X, \mathbb{A}}(N): X \rightarrow \mathbb{A},
$$

where

$$
\mathcal{I}_{X, \mathbb{A}}(f)(\sigma)= \begin{cases}\left\{f_{\tau, \rho}\right\}_{\rho \leqslant \tau \leqslant \sigma}: \sum_{\rho \leqslant \sigma} M(\rho) \rightarrow \sum_{\tau \leqslant \sigma} N(\tau), & \mathbb{A}(X)=\mathbb{A}^{*}(X), \\ \left\{f_{\tau, \rho}\right\}_{\sigma \leqslant \tau \leqslant \rho}: \sum_{\sigma \leqslant \rho} M(\rho) \rightarrow \sum_{\sigma \leqslant \tau} N(\tau), & \mathbb{A}(X)=\mathbb{A}_{*}(X) .\end{cases}
$$

Remark 4.15. There are a few notational differences worth highlighting:

- For a functor $C: X \rightarrow \mathbb{A}$ in $\mathbb{A}[X]$ we use the notation $C(\sigma)$ where Ranicki would write $C[\sigma]$.

- In [Ran92] the functor $\mathcal{I}_{X, \mathbb{A}}$ and its extension $\left(\mathcal{I}_{X, \mathbb{A}}\right)_{\mathbb{B}}$ are both denoted by square brackets, i.e. $\mathcal{I}_{X, \mathbb{A}}(M)=[M],\left(\mathcal{I}_{X, \mathbb{A}}\right)_{\mathbb{B}}(C)=[C]$. We prefer to distinguish $\mathcal{I}_{X, \mathbb{A}}$ from $\left(\mathcal{I}_{X, \mathbb{A}}\right)_{\mathbb{B}}$.

- Combining the above, $\mathcal{I}_{X, \mathbb{A}}(M)(\sigma)$ corresponds to $[M][\sigma]$ in [Ran92].

Example 4.16. The functors $\mathcal{I}_{X, \mathbb{A}}$ can be expressed using restriction and assembly functors as follows.

$$
\begin{aligned}
\mathcal{I}_{X, \mathbb{A}}(M)(\sigma) & =\left\{\begin{array}{l}
\operatorname{Ass}\left(\left.M\right|_{\sigma}\right), \\
\operatorname{Ass}\left(\left.M\right|_{\mathrm{st}(\sigma)}\right)
\end{array}\right. \\
\mathcal{I}_{X, \mathbb{A}}(M)(\tau \rightarrow \sigma) & =\left\{\begin{array}{l}
\operatorname{incl.}: \operatorname{Ass}\left(\left.M\right|_{\tau}\right) \hookrightarrow \operatorname{Ass}\left(\left.M\right|_{\sigma}\right) \\
\operatorname{incl.}: \operatorname{Ass}\left(\left.M\right|_{\mathrm{st}(\sigma)}\right) \hookrightarrow \operatorname{Ass}\left(\left.M\right|_{\mathrm{st}(\tau)}\right),
\end{array}\right. \\
\mathcal{I}_{X, \mathbb{A}}(f)(\sigma) & =\left\{\begin{array}{l}
\operatorname{Ass}\left(\left.f\right|_{\sigma}\right), \\
\operatorname{Ass}\left(\left.f\right|_{\mathrm{st}(\sigma)}\right)
\end{array}\right.
\end{aligned}
$$

in the case where

$$
\mathbb{A}(X)=\left\{\begin{array}{l}
\mathbb{A}^{*}(X), \\
\mathbb{A}_{*}(X),
\end{array}\right.
$$

where the open star st $(\sigma)$ denotes the simplicial pair $(\operatorname{St}(\sigma), \partial \operatorname{St}(\sigma))$ consisting of the closed star of $\sigma$ and its boundary.

Example 4.17. Let $D \in \mathbb{B}\left(\mathbb{A}(\mathbb{Z})_{*}(X)\right)$ be as in Example 4.11. It follows that

$$
\bigcup_{\tau \geqslant \sigma} D(\tau, X) \backslash \partial D(\tau, X)=D(\sigma, X)
$$

Then $\left(\mathcal{I}_{X, \mathbb{A}}\right)_{\mathbb{B}}(D)$ is the functor in $\mathbb{B}\left(\mathbb{A}(\mathbb{Z})_{*}[X]\right)=\mathbb{B}(\mathbb{A}(\mathbb{Z}))_{*}[X]$ given by $\left(\mathcal{I}_{X, \mathbb{A}}\right)_{\mathbb{B}}(D)(\sigma)=C_{*}(D(\sigma, X) ; \mathbb{Z})$, for all $\sigma \in X$ and such that

$$
\left(\mathcal{I}_{X, \mathbb{A}}\right)_{\mathbb{B}}(D)(\tau \rightarrow \sigma): C_{*}(D(\sigma, X) ; \mathbb{Z}) \rightarrow C_{*}(D(\tau, X) ; \mathbb{Z})
$$

is an inclusion map, for all $\tau \leqslant \sigma$. 
The tensor product $-\otimes_{\mathbb{A}}-=\operatorname{Hom}_{\mathbb{A}}\left(T_{\mathbb{B}}(-),-\right)$ and its switch natural isomorphism (see Proposition 3.10) together define a tensor product, also denoted $-\otimes_{\mathbb{A}}-$, on the functor categories $\mathbb{B}(\mathbb{A}[X])$ as follows.

Definition 4.18. Let $\left(T_{\mathbb{A}}, e_{\mathbb{A}}\right)$ be a chain duality on an additive category $\mathbb{A}$ with extensions $\left(T_{\mathbb{B}}, e_{\mathbb{B}}\right)$ as in Proposition 3.1. Following [Ran92, p.74], define tensor product functors

$$
\begin{aligned}
& -\otimes_{\mathbb{A}}-: \mathbb{B}\left(\mathbb{A}^{*}[X]\right) \times \mathbb{B}\left(\mathbb{A}^{*}[X]\right) \rightarrow \mathbb{B}(\mathbb{Z})^{*}[X], \\
& -\otimes_{\mathbb{A}}-: \mathbb{B}\left(\mathbb{A}_{*}[X]\right) \times \mathbb{B}\left(\mathbb{A}_{*}[X]\right) \rightarrow \mathbb{B}(\mathbb{Z})_{*}[X]
\end{aligned}
$$

by sending an object $(C, D)$ of $\mathbb{B}(\mathbb{A}[X]) \times \mathbb{B}(\mathbb{A}[X])$ to the object $C \otimes_{\mathbb{A}} D$ of $\mathbb{B}(\mathbb{Z})[X]$ given by

$$
\begin{aligned}
\left(C \otimes_{\mathbb{A}} D\right)(\sigma) & =C(\sigma) \otimes_{\mathbb{A}} D(\sigma)=\operatorname{Hom}_{\mathbb{A}}\left(T_{\mathbb{B}}(C(\sigma)), D(\sigma)\right), \\
\left(C \otimes_{\mathbb{A}} D\right)(\tau \rightarrow \sigma) & =C(\tau \rightarrow \sigma) \otimes_{\mathbb{A}} D(\tau \rightarrow \sigma)=T_{\mathbb{B}}(C(\tau \rightarrow \sigma))^{*}(D(\tau \rightarrow \sigma))_{*}
\end{aligned}
$$

and sending a morphism $\left(f: C \rightarrow D, f^{\prime}: C^{\prime} \rightarrow D^{\prime}\right)$ of $\mathbb{B}(\mathbb{A}[X]) \times \mathbb{B}(\mathbb{A}[X])$ to the morphism $f \otimes_{\mathbb{A}} f^{\prime}$ of $\mathbb{B}(\mathbb{Z})[X]$ given by

$$
\left(f \otimes_{\mathbb{A}} f^{\prime}\right)(\sigma)=T_{\mathbb{B}}(f(\sigma))^{*} f^{\prime}(\sigma)_{*}: C(\sigma) \otimes_{\mathbb{A}} C^{\prime}(\sigma) \rightarrow D(\sigma) \otimes_{\mathbb{A}} D^{\prime}(\sigma) .
$$

Proposition 4.19. For the tensor product $-\otimes_{\mathbb{A}}-$ of Definition 4.18, there are natural isomorphisms

$$
T_{-,-}:-\otimes_{\mathbb{A}}-\Rightarrow\left(-\otimes_{\mathbb{A}}-\right) \circ \mathcal{S}_{\mathbb{B}(\mathbb{A}[X])},
$$

with $T_{C, D}: C \otimes_{\mathbb{A}} D \stackrel{\cong}{\longrightarrow} D \otimes_{\mathbb{A}} C$ the chain isomorphism defined by

$$
T_{C, D}(\sigma)=T_{C(\sigma), D(\sigma)}: C(\sigma) \otimes_{\mathbb{A}} D(\sigma) \rightarrow D(\sigma) \otimes_{\mathbb{A}} C(\sigma),
$$

where $T_{C(\sigma), D(\sigma)}=\left(\operatorname{id}_{D(\sigma)} \otimes_{\mathbb{A}} e_{\mathbb{B}}(C(\sigma))\right) \circ T_{\mathbb{B}}$ as in Proposition 3.8.

Proof. This follows directly from Definition 4.18, naturality of $T_{\mathbb{B}}$ (Proposition 3.7) and naturality of $e_{\mathbb{B}}$.

A chain duality can also be applied componentwise to switch between upper and lower star categories as follows.

Proposition 4.20. A chain duality $\left(T_{\mathbb{A}}, e_{\mathbb{A}}\right)$ on $\mathbb{A}$ and its extension $\left(T_{\mathbb{B}}, e_{\mathbb{B}}\right)$ to $\mathbb{B}(\mathbb{A})$ induce functors

$$
\begin{aligned}
& \mathcal{T}_{\mathbb{A}}: \mathbb{A}_{*}(X) \rightarrow \mathbb{B}\left(\mathbb{A}^{*}(X)\right), \\
& \mathcal{T}_{\mathbb{A}}: \mathbb{A}^{*}(X) \rightarrow \mathbb{B}\left(\mathbb{A}_{*}(X)\right),
\end{aligned}
$$

with extensions $\mathcal{T}_{\mathbb{B}}$ and equivalences of functors

$$
\begin{aligned}
& \epsilon_{\mathbb{A}}: \mathcal{T}_{\mathbb{B}} \circ \mathcal{T}_{\mathbb{A}} \Rightarrow \iota_{\mathbb{A}(X)}: \mathbb{A}(X) \rightarrow \mathbb{B}(\mathbb{A}(X)), \\
& \epsilon_{\mathbb{B}}: \mathcal{T}_{\mathbb{B}} \circ \mathcal{T}_{\mathbb{B}} \Rightarrow \operatorname{id}_{\mathbb{B}(\mathbb{A}(X))}: \mathbb{B}(\mathbb{A}(X)) \rightarrow \mathbb{B}(\mathbb{A}(X)) .
\end{aligned}
$$

Proof. The functors $\mathcal{T}_{\mathbb{A}}$ are defined by applying $T_{\mathbb{A}}$ componentwise to objects and morphisms. Similarly, $\epsilon_{\mathbb{A}}$ is $e_{\mathbb{A}}$ applied componentwise. Extensions work by Proposition 3.1. 
Definition 4.21. Define tensor product functors

$$
\begin{aligned}
& -\otimes_{\mathbb{Z}}-: \mathbb{A}(\mathbb{Z}) \times \mathbb{A} \rightarrow \mathbb{A}, \\
& -\otimes_{\mathbb{Z}}-: \mathbb{A} \times \mathbb{A}(\mathbb{Z}) \rightarrow \mathbb{A}
\end{aligned}
$$

as follows. For all $\mathbb{Z}^{n} \in \mathbb{A}(\mathbb{Z}), M \in \mathbb{A}$ let

$$
\mathbb{Z}^{n} \otimes_{\mathbb{Z}} M=M \otimes_{\mathbb{Z}} \mathbb{Z}^{n}=M^{n}=\underbrace{M \oplus \cdots \oplus M}_{n \text { copies }} .
$$

For all morphisms $f: M \rightarrow N$ in $\mathbb{A}$ and $g: \mathbb{Z}^{m} \rightarrow \mathbb{Z}^{n}$ in $\mathbb{A}(\mathbb{Z})$ let

$$
g \otimes_{\mathbb{Z}} f=f \otimes_{\mathbb{Z}} g: M^{m} \rightarrow N^{n}
$$

be the morphism with $(i, j)^{\text {th }}$ component

$$
\left(g \otimes_{\mathbb{Z}} f\right)_{i j}=\left(f \otimes_{\mathbb{Z}} g\right)_{i j}=\underbrace{f+\cdots+f}_{g_{i j} \text { times }}: M \rightarrow N .
$$

These extend to bounded chain complexes in the usual way.

The following Proposition defines a new tensor product between round and square bracket categories. This tensor product gives rise to a simple interpretation of Ranicki's functor $T_{\mathbb{A}(X)}$ (cf. [Ran92, Proposition 5.1]) for $X$ a finite simplicial complex. Extending this to a ball complex $X$, this tensor product is also instrumental in proving that $\left(T_{\mathbb{A}(X)}, e_{\mathbb{A}(X)}\right)$ is a chain duality.

Proposition 4.22. Let $\mathbb{A}$ be an additive category and $X$ a ball complex. Let $\mathcal{C}$ be either $\mathbb{A}$ or $\mathbb{A}(\mathbb{Z})$ and $\mathcal{D}$ the other of the two. Then there are tensor product functors

$$
\begin{aligned}
& -\otimes-: \mathbb{B}\left(\mathcal{C}^{*}(X)\right) \times \mathbb{B}(\mathcal{D})_{*}[X] \rightarrow \mathbb{B}\left(\mathbb{A}^{*}(X)\right), \\
& -\otimes-: \mathbb{B}\left(\mathcal{C}_{*}(X)\right) \times \mathbb{B}(\mathcal{D})^{*}[X] \rightarrow \mathbb{B}\left(\mathbb{A}_{*}(X)\right)
\end{aligned}
$$

defined as follows.

For a pair of objects $(C, D) \in\left\{\begin{array}{l}\mathbb{B}\left(\mathcal{C}^{*}(X)\right) \times \mathbb{B}(\mathcal{D})_{*}[X], \quad \text { let } C \otimes D \text { be the object } \\ \mathbb{B}\left(\mathcal{C}_{*}(X)\right) \times \mathbb{B}(\mathcal{D})^{*}[X],\end{array}\right.$ in $\left\{\begin{array}{l}\mathbb{B}\left(\mathbb{A}^{*}(X)\right), \\ \mathbb{B}\left(\mathbb{A}_{*}(X)\right),\end{array}\right.$ with $n$-chains given by

$$
(C \otimes D)_{n}(\sigma)=\sum_{p+q=n} C_{p}(\sigma) \otimes_{\mathbb{Z}} D(\sigma)_{q},
$$

for $-\otimes_{\mathbb{Z}}-$ the tensor product of Definition 4.21 and differential

$$
\left(\left(d_{C \otimes D}\right)_{n}\right)_{\tau, \sigma}:(C \otimes D)_{n}(\sigma) \rightarrow(C \otimes D)_{n-1}(\tau)
$$

given by

$$
\left\{\begin{array}{l}
\sum_{p+q=n}\left(\left(\left(d_{C}\right)_{p}\right)_{\tau, \sigma} \otimes_{\mathbb{Z}} D(\tau \rightarrow \sigma)_{q}+(-1)^{p}\left(\operatorname{id}_{C_{p}}\right)_{\tau, \sigma} \otimes_{\mathbb{Z}}\left(d_{D(\sigma)}\right)_{q}\right), \\
\sum_{p+q=n}\left(\left(\left(d_{C}\right)_{p}\right)_{\tau, \sigma} \otimes_{\mathbb{Z}} D(\sigma \rightarrow \tau)_{q}+(-1)^{p}\left(\operatorname{id}_{C_{p}}\right)_{\tau, \sigma} \otimes_{\mathbb{Z}}\left(d_{D(\sigma)}\right)_{q}\right) .
\end{array}\right.
$$

For a pair $\left(f: C \rightarrow D, f^{\prime}: C^{\prime} \Rightarrow D^{\prime}\right)$ of morphisms of

$$
\left\{\begin{array}{l}
\mathbb{B}\left(\mathcal{C}^{*}(X)\right) \times \mathbb{B}(\mathcal{D})_{*}[X] \\
\mathbb{B}\left(\mathcal{C}_{*}(X)\right) \times \mathbb{B}(\mathcal{D})^{*}[X]
\end{array}\right.
$$


the morphism $f \otimes f^{\prime}: C \otimes C^{\prime} \rightarrow D \otimes D^{\prime}$ of $\left\{\begin{array}{l}\mathbb{B}\left(\mathbb{A}^{*}(X)\right), \\ \mathbb{B}\left(\mathbb{A}_{*}(X)\right)\end{array}\right.$ is defined by

$$
\left(\left(f \otimes f^{\prime}\right)_{n}\right)_{\tau, \sigma}=\delta_{\tau \sigma} \sum_{p+q=n}\left(f_{p}\right)_{\sigma, \sigma} \otimes_{\mathbb{Z}} f^{\prime}(\sigma)_{q}:\left(C \otimes C^{\prime}\right)_{n}(\sigma) \rightarrow\left(D \otimes D^{\prime}\right)_{n}(\tau),
$$

where $\delta_{\tau \sigma}$ is a Kronecker delta function.

Proof. Straightforward.

Remark 4.23. One can of course also define tensor products as in Proposition 4.22 but with the order of categories in the domain switched. There are natural switch isomorphisms between the switched tensor products and those of Proposition 4.22. The chain isomorphisms $C \otimes D \cong D \otimes C$ use a sign of $(-1)^{p q}$ for the component sending $C_{p} \otimes_{\mathbb{Z}} D_{q}$ to $D_{q} \otimes_{\mathbb{Z}} C_{p}$.

Example 4.24. As in Example 4.5 of $[$ Ran92 $]$ let $\underline{\mathbb{Z}} \in\left\{\begin{array}{l}\mathbb{A}(\mathbb{Z})^{*}[X] \subset \mathbb{B}(\mathbb{A}(\mathbb{Z}))^{*}[X], \\ \mathbb{A}(\mathbb{Z})_{*}[X] \subset \mathbb{B}(\mathbb{A}(\mathbb{Z}))_{*}[X]\end{array}\right.$ denote the functor with $\underline{\mathbb{Z}}(\sigma)=\mathbb{Z}$ for all $\sigma \in X$ and $\underline{\mathbb{Z}}(\tau \rightarrow \sigma)=\operatorname{id}_{\mathbb{Z}}$ for all $\tau \leqslant \sigma$.

Then $-\otimes \underline{\mathbb{Z}}$ is seen to be the identity functor on $\left\{\begin{array}{l}\mathbb{B}\left(\mathbb{A}_{*}(X)\right), \\ \mathbb{B}\left(\mathbb{A}^{*}(X)\right)\end{array}\right.$ by examining the formulae of Proposition 4.22. As $\underline{\mathbb{Z}}(\sigma)_{q}$ is $\mathbb{Z}$ when $q=0$ and 0 otherwise it follows that

$$
(C \otimes \underline{\mathbb{Z}})_{n}(\sigma)=\sum_{p+q=n} C_{p}(\sigma) \otimes_{\mathbb{Z}} \underline{\mathbb{Z}}(\sigma)_{q}=C_{n}(\sigma) \otimes_{\mathbb{Z}} \mathbb{Z}=C_{n}(\sigma) .
$$

As $d_{\underline{Z}(\sigma)}=0$ for all $\sigma \in X$ the second term in (4.1) is always zero. As $D(\tau \rightarrow \sigma)_{q}$ is $i d_{\mathbb{Z}}$ when $q=0$ and $\left\{\begin{array}{l}\tau \leqslant \sigma, \\ \sigma \leqslant \tau\end{array}\right.$ and 0 otherwise it follows that the first term agrees with the differential of the original chain complex $C$.

Another important chain complex to tensor with is $C(X)$, the $\mathbb{Z}$-coefficient cellular chain or cochain complex of $X$.

Definition 4.25. Define the shift functors

$$
\begin{aligned}
& \text { sh: } \mathbb{B}(\mathbb{A})^{*}[X] \rightarrow \mathbb{B}\left(\mathbb{A}_{*}(X)\right), \\
& \text { sh: } \mathbb{B}(\mathbb{A})_{*}[X] \rightarrow \mathbb{B}\left(\mathbb{A}^{*}(X)\right)
\end{aligned}
$$

as the functors that tensor on the left with the chain complex $C(X)$ of Definition 4.12 using the tensor products of Proposition 4.22, i.e. $\mathrm{sh}=C(X) \otimes-$.

Example 4.26. We see that

with differential

$$
\operatorname{sh}(C)_{n}(\sigma)=\left\{\begin{array}{l}
C(\sigma)_{n+|\sigma|}, \\
C(\sigma)_{n-|\sigma|},
\end{array}\right.
$$

$$
\left(\left(d_{\operatorname{sh}(C)}\right)_{n}\right)_{\tau, \sigma}=\left\{\begin{array}{c}
\left(\left(d_{C^{-*}(X ; \mathbb{Z})}\right)_{-|\sigma|}\right)_{\tau, \sigma} \otimes_{\mathbb{Z}} C(\sigma \rightarrow \tau)_{n+|\sigma|} \\
+(-1)^{-|\sigma|}\left(\left(\operatorname{id}_{C^{-*}(X ; \mathbb{Z})}\right)_{-|\sigma|}\right)_{\tau, \sigma} \otimes_{\mathbb{Z}}\left(d_{C(\sigma)}\right)_{n+|\sigma|}, \\
\left(\left(d_{\left.\left.C_{*}(X ; \mathbb{Z})\right)_{|\sigma|}\right)_{\tau, \sigma}} \otimes_{\mathbb{Z}} C(\tau \rightarrow \sigma)_{n-|\sigma|}\right.\right. \\
+(-1)^{|\sigma|}\left(\left(\operatorname{id}_{C_{*}(X ; \mathbb{Z})}\right)_{|\sigma|}\right)_{\tau, \sigma} \otimes_{\mathbb{Z}}\left(d_{C(\sigma)}\right)_{n-|\sigma|},
\end{array}\right.
$$

for $C \in\left\{\begin{array}{l}\mathbb{B}(\mathbb{A})^{*}[X], \\ \mathbb{B}(\mathbb{A})_{*}[X] .\end{array}\right.$ 
Morphisms are shifted similarly:

$$
\left(\operatorname{sh}(f)_{n}\right)_{\tau, \sigma}= \begin{cases}\left(\left(\operatorname{id}_{C^{-*}(X ; \mathbb{Z})}\right)_{-|\sigma|}\right)_{\tau, \sigma} \otimes_{\mathbb{Z}} f(\sigma)_{n+|\sigma|}, & f \in \operatorname{Mor}\left(\mathbb{B}(\mathbb{A})^{*}[X]\right), \\ \left(\left(\operatorname{id}_{C_{*}(X ; \mathbb{Z})}\right)_{|\sigma|}\right)_{\tau, \sigma} \otimes_{\mathbb{Z}} f(\sigma)_{n-|\sigma|}, & f \in \operatorname{Mor}\left(\mathbb{B}(\mathbb{A})^{*}[X]\right) .\end{cases}
$$

Comparing these formulae to Definition 4.4 of [Ran92] we see that the shift functors are precisely the same as Ranicki's covariant assembly functors in the case that $X$ is a simplicial complex. ${ }^{1}$ Combining this with Example 4.24 recovers the statement in [Ran92, Example 4.5] that the covariant assembly of $\underline{\mathbb{Z}}$ is $C(X)$

\section{Chain duality on categories over ball complexes}

Let $X$ be a ball complex, $\mathbb{A}$ an additive category and $\left(T_{\mathbb{A}}, e_{\mathbb{A}}\right)$ a chain duality on $\mathbb{A}$. We follow the approach in Section 3 to define a chain duality on $\mathbb{A}_{*}(X)$ and $\mathbb{A}^{*}(X)$ using $\left(T_{\mathbb{A}}, e_{\mathbb{A}}\right)$.

The following definition can be seen to agree with that of Proposition 5.1 of [Ran92] in the case that $X$ is a simplicial complex.

Definition 5.1. Let $\left(T_{\mathbb{A}}, e_{\mathbb{A}}\right)$ be a chain duality on an additive category $\mathbb{A}$. Define the contravariant functor $T_{\mathbb{A}(X)}: \mathbb{A}(X) \rightarrow \mathbb{B}(\mathbb{A}(X))$ to be the composition

$$
T_{\mathbb{A}(X)}=\operatorname{sh} \circ\left(T_{\mathbb{A}}\right)_{*} \circ \mathcal{I}_{X, \mathbb{A}},
$$

where $\left(T_{\mathbb{A}}\right)_{*}$ is the push-forward functor as defined in Definition 4.3.

Example 5.2. Let $X$ be a ball complex with $\rho \in X$ fixed. Suppose $C \in\left\{\begin{array}{l}\mathbb{B}\left(\mathbb{A}^{*}(X)\right) \text {, } \\ \mathbb{B}\left(\mathbb{A}_{*}(X)\right)\end{array}\right.$ is such that $C(\tau) \neq 0$ if and only if $\tau=\rho$. Then unpacking Definition 5.1 and using Example 4.17 it follows that

$$
\begin{aligned}
T_{\mathbb{B}(\mathbb{A}(X))}(C)(\sigma) & =\left\{\begin{array}{l}
C_{*}(\sigma, \partial \sigma ; \mathbb{Z}) \otimes_{\mathbb{Z}} T_{\mathbb{B}}\left(\left(I_{X, \mathbb{A}}\right)_{\mathbb{B}}(C(\sigma))\right) \\
C^{-*}(\sigma, \partial \sigma ; \mathbb{Z}) \otimes_{\mathbb{Z}} T_{\mathbb{B}}\left(\left(I_{X, \mathbb{A}}\right)_{\mathbb{B}}(C(\sigma))\right)
\end{array}\right. \\
& = \begin{cases}\Sigma^{|\sigma|} T_{\mathbb{B}}\left(\left.C\right|_{\sigma}\right) \\
\Sigma^{-|\sigma|} T_{\mathbb{B}}\left(\left.C\right|_{\mathrm{st}(\sigma)}\right)\end{cases} \\
& = \begin{cases}\Sigma^{|\sigma|} T_{\mathbb{B}}(C(\rho)), & \text { if } \sigma \geqslant \rho, \\
\Sigma^{-|\sigma|} T_{\mathbb{B}}(C(\rho)), & \text { if } \sigma \leqslant \rho,\end{cases}
\end{aligned}
$$

and 0 otherwise.

Example 5.3. For the ring of integers $R=\mathbb{Z}$ let $(T, e)$ be the chain duality on $\mathbb{A}(\mathbb{Z})$ defined in Example 3.3. Let $D \in \mathbb{B}\left(\mathbb{A}(\mathbb{Z})_{*}(X)\right)$ be as in Examples 4.11 and 4.17.

By Example 4.17 we have that $\left(\mathcal{I}_{X, \mathbb{A}}\right)_{\mathbb{B}}(D)(\sigma)=C_{*}(D(\sigma, X) ; \mathbb{Z})$, for all $\sigma \in X$. Therefore it follows that

$$
\begin{aligned}
T_{\mathbb{B}\left(\mathbb{A}(\mathbb{Z})_{*}(X)\right)}(D)(\sigma) & =\Sigma^{-|\sigma|} T\left(C_{*}(D(\sigma, X) ; \mathbb{Z})\right) \\
& =C^{-|\sigma|-*}(D(\sigma, X) ; \mathbb{Z}) .
\end{aligned}
$$

\footnotetext{
${ }^{1}$ Modulo possibly a different sign convention for the differential of the total complex of a double complex.
} 
In (3.1) a tensor product is defined for an additive category $\mathbb{A}$ with chain duality $\left(T_{\mathbb{A}}, e_{\mathbb{A}}\right)$ where, for objects $M, N \in \mathbb{A}, M \otimes_{\mathbb{A}} N$ is just a chain complex of Abelian groups. This definition only requires the contravariant functor part of the chain duality. Applying this to the categories $\mathbb{A}(X)$ and the contravariant functors $T_{\mathbb{A}(X)}$ of Definition 5.1 gives a tensor product over $\mathbb{A}(X)$. In the following it will be necessary to use a refinement of this tensor product where the tensor product of two objects $M, N \in \mathbb{A}(X)$ is not just a chain complex of Abelian groups but rather is fragmented over the ball complex $X$ in the sense of the previous section.

Definition 5.4. The contravariant functors $T_{\mathbb{A}(X)}: \mathbb{A}(X) \rightarrow \mathbb{B}(\mathbb{A}(X))$ and their extensions $T_{\mathbb{B}(\mathbb{A}(X))}=\left(T_{\mathbb{A}(X)}\right)_{\mathbb{B}}: \mathbb{B}(\mathbb{A}(X)) \rightarrow \mathbb{B}(\mathbb{A}(X))$ are used to define tensor product functors

$$
\begin{aligned}
& -\otimes_{\mathbb{A}^{*}(X)}-=\operatorname{Hom}_{\mathbb{A}^{*}(X)}\left(T_{\mathbb{B}\left(\mathbb{A}^{*}(X)\right)}(-),-\right): \mathbb{B}\left(\mathbb{A}^{*}(X)\right) \times \mathbb{B}\left(\mathbb{A}^{*}(X)\right) \rightarrow \mathbb{B}\left(\mathbb{Z}_{*}(X)\right), \\
& -\otimes_{\mathbb{A}_{*}(X)}-=\operatorname{Hom}_{\mathbb{A}_{*}(X)}\left(T_{\mathbb{B}\left(\mathbb{A}_{*}(X)\right)}(-),-\right): \mathbb{B}\left(\mathbb{A}_{*}(X)\right) \times \mathbb{B}\left(\mathbb{A}_{*}(X)\right) \rightarrow \mathbb{B}\left(\mathbb{Z}^{*}(X)\right),
\end{aligned}
$$

by

$$
\begin{aligned}
& \left(C \otimes_{\mathbb{A}^{*}(X)} D\right)_{n}(\sigma)=\operatorname{Hom}_{\mathbb{A}}\left(T_{\mathbb{B}\left(\mathbb{A}^{*}(X)\right)}(C)(\sigma),\left(\mathcal{I}_{X, \mathbb{A}}\right)_{\mathbb{B}}(D)(\sigma)\right)_{n}, \\
& \left(d_{C \otimes_{\mathbb{A}^{*}(X)} D}\right)_{n, \tau, \sigma}=(-1)^{n-1}\left(d_{T_{\mathbb{B}\left(\mathbb{A}^{*}(X)\right)}(C)}\right)_{\sigma, \tau}^{*}\left(\left(\mathcal{I}_{X, \mathbb{A}}\right)_{\mathbb{B}}(D)(\tau \rightarrow \sigma)\right)_{*} \\
& +\left(\operatorname{id}_{T_{\mathbb{B}\left(\mathbb{A}^{*}(X)\right)}(C)}\right)_{\sigma, \tau}^{*}\left(\left(\mathcal{I}_{X, \mathbb{A}}\right)_{\mathbb{B}}\left(d_{D}\right)(\sigma)\right)_{*}, \\
& \left(f \otimes_{\mathbb{A}^{*}(X)} f^{\prime}\right)_{n, \tau, \sigma}=T_{\mathbb{B}\left(\mathbb{A}^{*}(X)\right)}(f)_{\sigma, \tau}^{*}\left(\left(\mathcal{I}_{X, \mathbb{A}}\right)_{\mathbb{B}}\left(f^{\prime}\right)(\sigma)\right)_{*}
\end{aligned}
$$

and similarly for $\mathbb{A}_{*}(X)$.

Remark 5.5. The formulae of Definition 5.4 are exactly what you get by replacing $\mathbb{A}$ with $\mathbb{A}(X)$ in $(3.1)$ and grouping things accordingly, i.e. tensor products $-\otimes_{\mathbb{A}(X)}-$ of Definition 5.4 are refinements which assemble to give the tensor products of (3.1).

The following proposition applied to objects is stated in the proof of [Ran92, Proposition 5.1]. Due to different sign conventions this is stated as an equality in [Ran92]. This proposition also indicates the reason for wanting to work with the refined tensor products of Definition 5.4.

Proposition 5.6. There are natural isomorphisms of functors

$$
\begin{aligned}
& -\otimes_{\mathbb{A}^{*}(X)}-\Rightarrow \operatorname{sh} \circ\left(-\otimes_{\mathbb{A}}-\right) \circ\left(\left(\mathcal{I}_{X, \mathbb{A}}\right)_{\mathbb{B}} \times\left(\mathcal{I}_{X, \mathbb{A}}\right)_{\mathbb{B}}\right), \\
& -\otimes_{\mathbb{A}_{*}(X)}-\Rightarrow \operatorname{sh} \circ\left(-\otimes_{\mathbb{A}}-\right) \circ\left(\left(\mathcal{I}_{X, \mathbb{A}}\right)_{\mathbb{B}} \times\left(\mathcal{I}_{X, \mathbb{A}}\right)_{\mathbb{B}}\right) .
\end{aligned}
$$

Proof. It can be shown that there are isomorphisms

$$
\Phi_{C, D}:\left(C \otimes_{\mathbb{A}(X)} D\right)_{n} \cong\left(\operatorname{sh}\left(\left(\mathcal{I}_{X, \mathbb{A}}\right)_{\mathbb{B}}(C) \otimes_{\mathbb{A}}\left(\mathcal{I}_{X, \mathbb{A}}\right)_{\mathbb{B}}(D)\right)\right)_{n},
$$

for all $C, D \in \mathbb{B}(\mathbb{A}(X))$. The differentials of source and target agree up to a sign which can be compensated for by choosing the correct signs for the components of $\Phi_{C, D}$. Modulo this isomorphism we have equality

$$
f \otimes_{\mathbb{A}(X)} f^{\prime}=\operatorname{sh}\left(\left(\mathcal{I}_{X, \mathbb{A}}\right)_{\mathbb{B}}(f) \otimes_{\mathbb{A}}\left(\mathcal{I}_{X, \mathbb{A}}\right)_{\mathbb{B}}\left(f^{\prime}\right)\right),
$$

for all morphisms $f, f^{\prime}$ in $\mathbb{B}(\mathbb{A}(X))$. This proves naturality. 
Proposition 5.7. There is a natural isomorphism

$$
T_{-,-}:-\otimes_{\mathbb{A}(X)}-\Rightarrow\left(-\otimes_{\mathbb{A}(X)}-\right) \circ \mathcal{S}_{\mathbb{B}(\mathbb{A}(X))},
$$

with $T_{C, D}: C \otimes_{\mathbb{A}(X)} D \stackrel{\cong}{\longrightarrow} D \otimes_{\mathbb{A}(X)} C$ defined as the composition

$$
T_{C, D}=\Phi_{D, C}^{-1} \circ \operatorname{sh}\left(T_{\left(\mathcal{I}_{X, \mathbb{A}}\right)_{\mathbb{B}}(C),\left(\mathcal{I}_{X, \mathbb{A}}\right)_{\mathbb{B}}(D)}\right) \circ \Phi_{C, D},
$$

where $\Phi_{C, D}$ and $\Phi_{D, C}^{-1}$ are isomorphisms as in the proof of Proposition 5.6 and $T_{\left(\mathcal{I}_{X, \mathbb{A}}\right)_{\mathbb{B}}(C),\left(\mathcal{I}_{X, \mathbb{A}}\right)_{\mathbb{B}}(D)}$ is as defined in Proposition 4.19.

As $T_{\left(\mathcal{I}_{X, \mathbb{A}}\right)_{\mathbb{B}}(D),\left(\mathcal{I}_{X, \mathbb{A}}\right)_{\mathbb{B}}(C)}=T_{\left(\mathcal{I}_{X, \mathbb{A}}\right)_{\mathbb{B}}(C),\left(\mathcal{I}_{X, \mathbb{A}}\right)_{\mathbb{B}}(D)}^{-1}$ for all $C, D \in \mathbb{B}(\mathbb{A}(X))$ it follows that $T_{D, C}=T_{C, D}^{-1}$ for all $C, D \in \mathbb{B}(\mathbb{A}(X))$.

Proof. For all $C, D \in \mathbb{B}(\mathbb{A}(X))$,

$$
T_{C, D}: C \otimes_{\mathbb{A}(X)} D \stackrel{\cong}{\longrightarrow} D \otimes_{\mathbb{A}(X)} C
$$

is a chain isomorphism as it is defined as the composition of three chain isomorphisms. The fact that $T_{-,-}$is a natural transformation follows from having natural transformations in Propositions 4.19 and 5.6.

Proposition 5.8. Let $e_{\mathbb{B}(\mathbb{A}(X))}(C)=T_{C, T_{\mathbb{B}(\mathbb{A}(X))}(C)}\left(\operatorname{id}_{T_{\mathbb{B}(\mathbb{A}(X))}(C)}\right)$, for all $C \in \mathbb{B}(\mathbb{A}(X))$. Then

1. this defines a natural transformation $e_{\mathbb{B}(\mathbb{A}(X))}: T_{\mathbb{B}(\mathbb{A}(X))}^{2} \Rightarrow \operatorname{id}_{\mathbb{B}(\mathbb{A}(X))}$,

2. $T_{C, D}(\phi)=e_{\mathbb{B}(\mathbb{A}(X))}(C) \circ T_{\mathbb{B}(\mathbb{A}(X))}(\phi)$, for all chain maps $\phi: T_{\mathbb{B}(\mathbb{A}(X))}(C) \rightarrow D$,

3. $e_{\mathbb{B}(\mathbb{A}(X))}\left(T_{\mathbb{B}(\mathbb{A}(X))}(C)\right) \circ T_{\mathbb{B}(\mathbb{A}(X))}\left(e_{\mathbb{B}(\mathbb{A}(X))}(C)\right)=\operatorname{id}_{T_{\mathbb{B}(\mathbb{A}(X))}(C)}, \forall C \in \mathbb{B}(\mathbb{A}(X))$.

Proof. This is an immediate consequence of Proposition 3.11 applied to $\mathbb{A}=\mathbb{A}(X)$ which may be applied since $T_{D, C}=T_{C, D}^{-1}$, for all $C, D \in \mathbb{B}(\mathbb{A}(X))$. The proof of Proposition 3.11 is categorical and does not depend on the codomain $\mathbb{B}(\mathrm{Ab})$ of $-\otimes_{\mathbb{A}}-$. Therefore Proposition 3.11 still holds for $\mathbb{A}=\mathbb{A}(X)$ using the refined tensor products of Definition 5.4.

The rest of the section is devoted to proving that $e_{\mathbb{B}(\mathbb{A}(X))}(C): T_{\mathbb{B}(\mathbb{A}(X))}^{2}(C) \simeq C$, for all $C \in \mathbb{B}(\mathbb{A}(X))$. By Proposition 4.13 it is sufficient to show that

$$
e_{\mathbb{B}(\mathbb{A}(X))}(C)_{\sigma, \sigma}: T_{\mathbb{B}(\mathbb{A}(X))}^{2}(C)(\sigma) \rightarrow C(\sigma)
$$

is a chain equivalence in $\mathbb{A}$, for all $\sigma \in X$.

Proposition 5.9. Let $e_{\mathbb{B}(\mathbb{A}(X))}: T_{\mathbb{B}(\mathbb{A}(X))}^{2} \Rightarrow \mathrm{id}_{\mathbb{B}(\mathbb{A}(X))}$ be as defined in Proposition 5.8. Then, for all $C \in \mathbb{B}(\mathbb{A}(X))$ and all $\sigma \in X$, the map

$$
e_{\mathbb{B}(\mathbb{A}(X))}(C)_{\sigma, \sigma}: T_{\mathbb{B}(\mathbb{A}(X))}^{2}(C)(\sigma) \rightarrow C(\sigma)
$$

is the composition of a signed projection map

$$
T_{\mathbb{B}(\mathbb{A}(X))}^{2}(C)(\sigma) \rightarrow T_{\mathbb{B}}^{2}(C(\sigma))
$$

and

$$
e_{\mathbb{B}}(C(\sigma)): T_{\mathbb{B}}^{2}(C(\sigma)) \rightarrow C(\sigma) .
$$


Proof. Applying $T_{C, D}=\Phi_{D, C}^{-1} \circ \operatorname{sh}\left(T_{\left(\mathcal{I}_{X, \mathbb{A}}\right)_{\mathbb{B}}(C),\left(\mathcal{I}_{X, \mathbb{A}}\right)_{\mathbb{B}}(D)}\right) \circ \Phi_{C, D}$ in the case where $D=T_{\mathbb{B}(\mathbb{A}(X))}(C)$ to

$$
\phi=\mathrm{id}_{T_{\mathbb{B}(\mathbb{A}(X))}(C)} \in\left(C \otimes_{\mathbb{A}(X)} T_{\mathbb{B}(\mathbb{A}(X))}(C)\right)_{0},
$$

the components

$$
e_{\mathbb{B}(\mathbb{A}(X))}(C)(\sigma)_{p, q}^{r}: T_{\mathbb{A}(X)}\left(T_{\mathbb{B}(\mathbb{A}(X))}(C)_{p}(\sigma)\right)_{q} \rightarrow\left(\mathcal{I}_{X, \mathbb{A}}\right)_{\mathbb{B}}(C)(\sigma)_{r}
$$

of $e_{\mathbb{B}(\mathbb{A}(X))}(C)=T_{C, T_{\mathbb{B}(\mathbb{A}(X))}(C)}(\phi)$ can be calculated.

Using the signs for $\Phi_{C, D}, \Phi_{D, C}$ of Proposition 5.6 together with (3.3) and Proposition 4.19 the components $e_{\mathbb{B}(\mathbb{A}(X))}(C)(\sigma)_{p, q}^{r}$ are computed to be

$$
(-1)^{|\sigma|(q-|\sigma|)}(-1)^{\frac{1}{2}|\sigma|(|\sigma|-1)} e_{\mathbb{A}}\left(\left(\mathcal{I}_{X, \mathbb{A}}\right)_{\mathbb{B}}(C)(\sigma)_{r}\right)_{q-|\sigma|, q-|\sigma|} \circ T_{\mathbb{A}}\left(\phi(\sigma)_{r, q}^{p}\right)_{q-|\sigma|},
$$

for all $p-q+r=0$ where $\phi(\sigma)_{r, q}^{p}$ is the inclusion map

$$
T_{\mathbb{B}(\mathbb{A}(X))}\left(C_{r}\right)_{q}(\sigma) \hookrightarrow\left(\mathcal{I}_{X, \mathbb{A}}\right)_{\mathbb{B}}\left(T_{\mathbb{B}(\mathbb{A}(X))}(C)_{p}\right)(\sigma) .
$$

Examining (5.1) and using additivity of $e_{\mathbb{A}}$ it follows that

$$
e_{\mathbb{B}(\mathbb{A}(X))}(C)_{\sigma, \sigma}= \pm e_{\mathbb{B}}(C(\sigma)) \circ \text { proj. }: T_{\mathbb{B}(\mathbb{A}(X))}^{2}(C)(\sigma) \rightarrow T_{\mathbb{B}}^{2}(C(\sigma)) \rightarrow C(\sigma)
$$

as required.

Consequently, since $e_{\mathbb{B}}(C(\sigma)): T_{\mathbb{B}}^{2}(C(\sigma)) \rightarrow C(\sigma)$ is a chain equivalence in $\mathbb{A}$, for all $\sigma \in X$, it is now sufficient to prove that the projection map

$$
T_{\mathbb{B}(\mathbb{A}(X))}^{2}(C)(\sigma) \rightarrow T_{\mathbb{B}}^{2}(C(\sigma))
$$

of Proposition 5.9 is a chain equivalence in $\mathbb{A}$. The signed projection map is a chain equivalence if and only if the unsigned projection map is; we prove this but must first make the following definitions.

Definition 5.10. For all $\sigma \in X$, define $\mathcal{D}_{*}^{\sigma}: X \rightarrow \mathbb{B}(\mathbb{A}(\mathbb{Z}))$ in $\mathbb{B}(\mathbb{A}(\mathbb{Z}))^{*}[X]$ by

$$
\begin{aligned}
\mathcal{D}_{*}^{\sigma}(\tau) & =C_{*}([\tau: \sigma] ; \mathbb{Z}), \\
\mathcal{D}_{*}^{\sigma}(\rho \rightarrow \tau) & =\text { restriction: } C_{*}([\rho: \sigma] ; \mathbb{Z}) \rightarrow C_{*}([\tau: \sigma] ; \mathbb{Z}) .
\end{aligned}
$$

Similarly define $\mathcal{D}_{\sigma}^{-*}: X \rightarrow \mathbb{B}(\mathbb{A}(\mathbb{Z}))$ in $\mathbb{B}(\mathbb{A}(\mathbb{Z}))_{*}[X]$ by

$$
\begin{aligned}
\mathcal{D}_{\sigma}^{-*}(\tau) & =C^{-*}([\sigma: \tau] ; \mathbb{Z}), \\
\mathcal{D}_{\sigma}^{-*}(\rho \rightarrow \tau) & =\text { restriction: } C^{-*}([\sigma: \tau] ; \mathbb{Z}) \rightarrow C^{-*}([\sigma: \rho] ; \mathbb{Z}) .
\end{aligned}
$$

Definition 5.11. For any $C \in \mathbb{B}(\mathbb{A})$, define the functor $C_{\sigma}: X \rightarrow \mathbb{B}(\mathbb{A})$ by

$$
C_{\sigma}(\tau)=\delta_{\tau \sigma} C(\sigma) \quad \text { and } \quad C_{\sigma}(\rho \rightarrow \tau)=\delta_{\rho \sigma} \delta_{\tau \sigma} \operatorname{id}_{C(\sigma)},
$$

where $\delta_{\tau \sigma}$ and $\delta_{\rho \sigma}$ are Kronecker $\delta$ functions. Note that $C_{\sigma}$ is in both $\mathbb{B}(\mathbb{A})^{*}[X]$ and $\mathbb{B}(\mathbb{A})_{*}[X]$ as it is supported on a single ball $\sigma \in X$.

Proposition 5.12. For all $\sigma \in X$, there are natural equivalences of functors

$$
e: \mathcal{D}_{*}^{\sigma} \Rightarrow\left(\Sigma^{|\sigma|} \mathbb{Z}\right)_{\sigma}, \quad e^{*}: \mathcal{D}_{\sigma}^{-*} \Rightarrow\left(\Sigma^{-|\sigma|} \mathbb{Z}\right)_{\sigma},
$$

in $\mathbb{B}(\mathbb{A}(\mathbb{Z}))^{*}[X]$ and $\mathbb{B}(\mathbb{A}(\mathbb{Z}))_{*}[X]$ respectively. 
Proof. By Proposition 2.4 there are chain contractions

$$
P_{\tau}: \mathcal{D}_{*}^{\sigma}(\tau) \simeq 0, \quad Q_{\tau}: \mathcal{D}_{\sigma}^{-*}(\tau) \simeq 0,
$$

for all $\tau \neq \sigma$ and

$$
\mathcal{D}_{*}^{\sigma}(\sigma)=\Sigma^{|\sigma|} \mathbb{Z}, \quad \mathcal{D}_{\sigma}^{-*}(\sigma)=\Sigma^{-|\sigma|} \mathbb{Z} .
$$

Define the natural transformations $e$ and $e^{*}$ by

$$
e(\tau)=\delta_{\tau \sigma} \operatorname{id}_{\Sigma^{|\sigma|} \mathbb{Z}} \quad \text { and } \quad e^{*}(\tau)=\delta_{\tau \sigma} \operatorname{id}_{\Sigma^{-|\sigma|} \mid \mathbb{Z}} .
$$

Checking these are natural transformations is straightforward and since

$$
e(\tau): \mathcal{D}_{*}^{\sigma}(\tau) \simeq\left(\Sigma^{|\sigma|} \mathbb{Z}\right)_{\sigma}(\tau) \text { and } e^{*}(\tau): \mathcal{D}_{\sigma}^{-*}(\tau) \simeq\left(\Sigma^{-|\sigma|} \mathbb{Z}\right)_{\sigma}(\tau)
$$

are chain equivalences for all $\tau \in X$ we see that $e$ and $e^{*}$ are natural equivalences.

Proposition 5.13. The projection maps $T_{\mathbb{B}(\mathbb{A}(X))}^{2}(C)(\sigma) \rightarrow T_{\mathbb{B}}^{2}(C(\sigma))$ of Proposition 5.9 are chain equivalences.

Proof. First observe that there are chain isomorphisms

$$
\begin{aligned}
&\left.T_{\mathbb{B}\left(\mathbb{A}^{*}(X)\right)}(C)\right|_{\sigma} \cong \\
&\left.T_{\mathbb{B}\left(\mathbb{A}_{*}(X)\right)}(C)\right|_{\operatorname{st}(\sigma)} \cong \mathcal{D}_{*} \otimes \mathcal{T}_{\mathbb{B}}(C), \\
& \mathcal{D}_{\sigma}^{-*} \otimes \mathcal{T}_{\mathbb{B}}(C),
\end{aligned}
$$

in $\mathbb{G}_{X}(\mathbb{A})$ given by redistributing summands on the left to different balls and redistributing morphisms to go between the new locations of the summands. For example,

$$
\left(\left.T_{\mathbb{B}\left(\mathbb{A}^{*}(X)\right)}(C)\right|_{\sigma}\right)_{n}(\tau)=\sum_{\rho \leqslant \tau \leqslant \sigma} T_{\mathbb{B}}(C(\rho))_{n-|\tau|},
$$

whereas

$$
\begin{aligned}
\left(\mathcal{D}_{*}^{\sigma} \otimes \mathcal{T}_{\mathbb{B}}(C)\right)_{n}(\rho) & =\left(C_{*}(\operatorname{st}(\rho) \cap \sigma ; \mathbb{Z}) \otimes_{\mathbb{Z}} T_{\mathbb{B}}(C(\rho))\right)_{n} \\
& =\sum_{\rho \leqslant \tau \leqslant \sigma} C_{|\tau|}(\tau, \partial \tau ; \mathbb{Z}) \otimes_{\mathbb{Z}} T_{\mathbb{B}}(C(\rho))_{n-|\tau|} \\
& =\sum_{\rho \leqslant \tau \leqslant \sigma} T_{\mathbb{B}}(C(\rho))_{n-|\tau|} .
\end{aligned}
$$

In this case the redistribution isomorphism takes the summand $T_{\mathbb{B}}(C(\rho))_{n-|\tau|}$ in $\left(\left.T_{\mathbb{B}\left(\mathbb{A}^{*}(X)\right)}(C)\right|_{\sigma}\right)_{n}$ associated to the ball $\tau$ and moves it to lie over $\rho$, which is where it is in $\left(\mathcal{D}_{*}^{\sigma} \otimes \mathcal{T}_{\mathbb{B}}(C)\right)_{n}$.

The natural equivalences of Proposition 5.12 induce chain equivalences

$$
\begin{aligned}
e \otimes \operatorname{id}_{\mathcal{T}_{\mathbb{B}}(C)}: \mathcal{D}_{*}^{\sigma} \otimes \mathcal{T}_{\mathbb{B}}(C) \stackrel{\simeq}{\longrightarrow}\left(\Sigma^{|\sigma|} \mathbb{Z}\right)_{\sigma} \otimes \mathcal{T}_{\mathbb{B}}(C), \\
e^{*} \otimes \operatorname{id}_{\mathcal{T}_{\mathbb{B}}(C)}: \mathcal{D}_{\sigma}^{-*} \otimes \mathcal{T}_{\mathbb{B}}(C) \stackrel{\simeq}{\longrightarrow}\left(\Sigma^{-|\sigma|} \mathbb{Z}\right)_{\sigma} \otimes \mathcal{T}_{\mathbb{B}}(C),
\end{aligned}
$$

in $\mathbb{A}(X)$. Further, since the chain equivalences are the identity map over $\sigma \in X$ and map to zero elsewhere they are projections onto the components supported on $\sigma$. The chain homotopy inverses are correspondingly inclusions of the components supported on $\sigma$. 
Assembling the above chain isomorphisms and chain equivalences gives chain equivalences in $\mathbb{B}(\mathbb{A})$ :

$$
\begin{aligned}
\operatorname{Ass}\left(\left.T_{\mathbb{B}\left(\mathbb{A}^{*}(X)\right)}(C)\right|_{\sigma}\right) & \cong \operatorname{Ass}\left(\mathcal{D}_{*}^{\sigma} \otimes \mathcal{T}_{\mathbb{B}}(C)\right) \\
& \cong \operatorname{css}\left(\left(\Sigma^{|\sigma|} \mathbb{Z}\right)_{\sigma} \otimes \mathcal{T}_{\mathbb{B}}(C)\right)=\Sigma^{|\sigma|} T_{\mathbb{B}}(C(\sigma)), \\
\operatorname{Ass}\left(\left.T_{\mathbb{B}\left(\mathbb{A}_{*}(X)\right)}(C)\right|_{\sigma}\right) & \cong \operatorname{\cong ss}\left(\mathcal{D}_{\sigma}^{-*} \otimes \mathcal{T}_{\mathbb{B}}(C)\right) \\
& \simeq \operatorname{Ass}\left(\left(\Sigma^{-|\sigma|} \mathbb{Z}\right)_{\sigma} \otimes \mathcal{T}_{\mathbb{B}}(C)\right)=\Sigma^{-|\sigma|} T_{\mathbb{B}}(C(\sigma)),
\end{aligned}
$$

which are also projections onto the component that used to be supported on $\sigma$ before assembly. Note that the chain homotopy inverses are the inclusions

$$
\begin{aligned}
\Sigma^{|\sigma|} T_{\mathbb{B}}(C(\sigma)) & \hookrightarrow \operatorname{Ass}\left(\left.T_{\mathbb{B}\left(\mathbb{A}^{*}(X)\right)}(C)\right|_{\sigma}\right), \\
\Sigma^{-|\sigma|} T_{\mathbb{B}}(C(\sigma)) & \hookrightarrow \operatorname{Ass}\left(\left.T_{\mathbb{B}\left(\mathbb{A}_{*}(X)\right)}(C)\right|_{\operatorname{st}(\sigma)}\right) .
\end{aligned}
$$

Applying $\Sigma^{|\sigma|} T_{\mathbb{B}}(-)$ and $\Sigma^{-|\sigma|} T_{\mathbb{B}}(-)$ to these inclusions give projection maps

$$
\begin{aligned}
T_{\mathbb{B}\left(\mathbb{A}^{*}(X)\right)}^{2}(C)(\sigma)=\Sigma^{|\sigma|} T_{\mathbb{B}}\left(\left.T_{\mathbb{B}\left(\mathbb{A}^{*}(X)\right)}(C)\right|_{\sigma}\right) \stackrel{\simeq}{\longrightarrow} \Sigma^{|\sigma|} T_{\mathbb{B}}\left(\Sigma^{|\sigma|} T_{\mathbb{B}}(C(\sigma))\right), \\
T_{\mathbb{B}\left(\mathbb{A}_{*}(X)\right)}^{2}(C)(\sigma)=\Sigma^{-|\sigma|} T_{\mathbb{B}}\left(\left.T_{\mathbb{B}\left(\mathbb{A}_{*}(X)\right)}(C)\right|_{\mathrm{st}(\sigma)}\right) \stackrel{\simeq}{\longrightarrow} \Sigma^{-|\sigma|} T_{\mathbb{B}}\left(\Sigma^{-|\sigma|} T_{\mathbb{B}}(C(\sigma))\right),
\end{aligned}
$$

which are also chain equivalences as $T_{\mathbb{B}}$ sends chain equivalences to chain equivalences by Proposition 3.6.

For a contravariant additive functor $\mathcal{G}: \mathbb{B}(\mathbb{A}) \rightarrow \mathbb{B}(\mathbb{A})$ it can be easily checked that there are isomorphisms of functors $\Sigma^{n} \circ \mathcal{G} \cong \mathcal{G} \circ \Sigma^{-n}$, for all $n \in \mathbb{Z}$. Consequently, we have isomorphisms

$$
\begin{aligned}
\Sigma^{|\sigma|} T_{\mathbb{B}}\left(\Sigma^{|\sigma|} T_{\mathbb{B}}(C(\sigma))\right) & \cong \Sigma^{|\sigma|} \Sigma^{-|\sigma|} T_{\mathbb{B}}^{2}(C(\sigma))=T_{\mathbb{B}}^{2}(C(\sigma)), \\
\Sigma^{-|\sigma|} T_{\mathbb{B}}\left(\Sigma^{-|\sigma|} T_{\mathbb{B}}(C(\sigma))\right) & \cong \Sigma^{-|\sigma|} \Sigma^{|\sigma|} T_{\mathbb{B}}^{2}(C(\sigma))=T_{\mathbb{B}}^{2}(C(\sigma)) .
\end{aligned}
$$

Composing the chain equivalences of all the previous steps shows that the projection maps $T_{\mathbb{B}(\mathbb{A}(X))}^{2}(C)(\sigma) \rightarrow T_{\mathbb{B}}^{2}(C(\sigma))$ are chain equivalences as required.

Example 5.14. Suppose $C \in \mathbb{B}^{*}(\mathbb{A}(X))$ is such that $C(\tau) \neq 0$ if and only if $\tau=\rho$. Then by Example 5.2

$$
T_{\mathbb{B}^{*}(\mathbb{A}(X))}(C)(\sigma)= \begin{cases}C_{*}(\sigma ; \partial \sigma ; \mathbb{Z}) \otimes_{\mathbb{Z}} T_{\mathbb{B}}(C(\rho)), & \sigma \geqslant \rho, \\ 0, & \text { otherwise. }\end{cases}
$$

The boundary maps are such that

$$
\operatorname{Ass}\left(T_{\mathbb{B}^{*}(\mathbb{A}(X))}(C)\right) \cong C_{*}(\operatorname{st}(\rho) ; \mathbb{Z}) \otimes_{\mathbb{Z}} T_{\mathbb{B}}(C(\rho)) .
$$

Restricting $T_{\mathbb{B}^{*}(\mathbb{A}(X))}(C)$ to $\sigma$, for any $\sigma \geqslant \rho$, the isomorphism (5.2) restricts to

$$
\operatorname{Ass}\left(\left.T_{\mathbb{B}^{*}(\mathbb{A}(X))}(C)\right|_{\sigma}\right) \cong C_{*}([\rho: \sigma] ; \mathbb{Z}) \otimes_{\mathbb{Z}} T_{\mathbb{B}}(C(\rho))=\operatorname{Ass}\left(\mathcal{D}_{*}^{\sigma} \otimes \mathcal{T}_{\mathbb{B}}(C)\right),
$$

where the equality in (5.3) follows from the fact that $\left(\mathcal{D}_{*}^{\sigma} \otimes \mathcal{T}_{\mathbb{B}}(C)\right)(\tau)$ is non-zero only for $\tau=\rho$ so that $\left(\mathcal{D}_{*}^{\sigma} \otimes \mathcal{T}_{\mathbb{B}}(C)\right)(\rho)=\operatorname{Ass}\left(\mathcal{D}_{*}^{\sigma} \otimes \mathcal{T}_{\mathbb{B}}(C)\right)$.

The isomorphism (5.3) is the total assembly of the redistribution isomorphism 
which maps

$$
C_{*}(\tau, \partial \tau ; \mathbb{Z}) \otimes_{\mathbb{Z}} T_{\mathbb{B}}(C(\rho))=T_{\mathbb{B}^{*}(\mathbb{A}(X))}(C)(\tau),
$$

for all $\rho \leqslant \tau \leqslant \sigma$ from the ball $\tau$ to the summand

$$
C_{*}(\tau, \partial \tau ; \mathbb{Z}) \otimes_{\mathbb{Z}} T_{\mathbb{B}}(C(\rho))=C_{*}(\tau, \partial \tau ; \mathbb{Z}) \otimes_{\mathbb{Z}} \mathcal{T}_{\mathbb{B}}(C)(\rho)
$$

of

$$
C_{*}([\rho: \sigma] ; \mathbb{Z}) \otimes_{\mathbb{Z}} \mathcal{T}_{\mathbb{B}}(C)(\rho)=\left(\mathcal{D}_{*}^{\sigma} \otimes \mathcal{T}_{\mathbb{B}}(C)\right)(\rho),
$$

which is associated to the ball $\rho$.

Next

$$
\begin{aligned}
T_{\mathbb{B}^{*}(\mathbb{A}(X))}^{2}(C)(\sigma) & =C_{*}(\sigma, \partial \sigma ; \mathbb{Z}) \otimes_{\mathbb{Z}} T_{\mathbb{B}}\left(\operatorname{Ass}\left(\left.T_{\mathbb{B}^{*}(\mathbb{A}(X))}(C)\right|_{\sigma}\right)\right) \\
& \cong \Sigma^{|\sigma|} T_{\mathbb{B}}\left(\operatorname{Ass}\left(\mathcal{D}_{*}^{\sigma} \otimes \mathcal{T}_{\mathbb{B}}(C)\right)\right) \\
& \simeq \Sigma^{|\sigma|} T_{\mathbb{B}}\left(\operatorname{Ass}\left(\left(\Sigma^{|\sigma|} \mathbb{Z}\right)_{\sigma} \otimes \mathcal{T}_{\mathbb{B}}(C)\right)\right) \\
& = \begin{cases}\Sigma^{|\sigma|} T_{\mathbb{B}}\left(\Sigma^{|\sigma|} T_{\mathbb{B}}(C(\rho))\right), & \sigma=\rho, \\
0, & \sigma \neq \rho .\end{cases}
\end{aligned}
$$

The last equality is due to $\left(\Sigma^{|\sigma|} \mathbb{Z}\right)_{\sigma}$ being 0 except over $\sigma$ and $\mathcal{T}_{\mathbb{B}}(C)$ being 0 except over $\rho$. Thus $\left(\Sigma^{|\sigma|} \mathbb{Z}\right)_{\sigma} \otimes \mathcal{T}_{\mathbb{B}}(C)$ is 0 except over $\sigma$ if $\sigma=\rho$ in which case it is equal to $\Sigma^{|\sigma|} T_{\mathbb{B}}(C(\rho))$. Hence we have

$$
T_{\mathbb{B}^{*}(\mathbb{A}(X))}^{2}(C)(\rho) \simeq \Sigma^{|\sigma|} T_{\mathbb{B}}\left(\Sigma^{|\sigma|} T_{\mathbb{B}}(C(\rho))\right) \cong T_{\mathbb{B}}^{2}(C(\rho))
$$

and

$$
T_{\mathbb{B}^{*}(\mathbb{A}(X))}^{2}(C)(\sigma) \simeq 0=T_{\mathbb{B}}^{2}(C(\sigma)),
$$

for $\sigma \neq \rho$. The $C \in \mathbb{B}_{*}(\mathbb{A}(X))$ case is similar.

\section{6. $\quad L$-homology theory}

Let us recall from $[\mathbf{R a n 9 2}]$ that, for an additive category with chain duality $\mathbb{A}$ and $n \in \mathbb{Z}$, we have the quadratic $L$-groups $L_{n}(\mathbb{A})$ [Ran92, Definition 1.8], the quadratic $L$-spaces $\mathbf{L}_{n}(\mathbb{A})[\mathbf{R a n 9 2}$, Definition 13.2] and the $L$-spectra $\mathbf{L} \bullet(\mathbb{A})[\mathbf{R a n 9 2}$, Proposition 13.4] defined using the notion of $n$-dimensional quadratic Poincaré complexes $(C, \psi)$ in $\mathbb{A}[$ Ran92, Definition 1.6]. Similarly there are symmetric versions with superscripts rather than subscripts.

Theorem 6.1. [Weig2] The collection of functors

$$
X \mapsto L_{n}\left(\mathbb{A}_{*}(X)\right)=\pi_{n}\left(\mathbf{L}_{\bullet}\left(\mathbb{A}_{*}(X)\right)\right)
$$

defines a homology theory on the category $\mathcal{S T} \mathcal{R C} \mathcal{E} \mathcal{L} \mathcal{L}$ of structured cell complexes.

Proof. Note that this functor factors through the forgetful functor $\mathcal{S T R C \mathcal { L } L} \rightarrow$ $\mathcal{B A L} \mathcal{L} \rightarrow \mathcal{R E G C W}$ and so we only need to show that this assignment is a homology theory on the category $\mathcal{R} \mathcal{E} \mathcal{G C W}$.

We closely follow [Wei92], where the same was proved with the category of finite $\Delta$-sets and inclusions as the source. The proofs turn out to apply in our situation basically verbatim. Note that only homotopy invariance and excision need to be proved. 
The existence of long exact sequences is automatic since the functors above factor through spectra. The key Lemma 3.1 from [Wei92] has in our context the same proof using the technology of localisation sequences in $L$-theory as in [Wei92, Theorem 2.3] or [Ran92, Proposition 13.11]. For homotopy invariance the same spectral sequence argument as in [Wei92, Corollary 3.2] can be used. The excision is the statement of [Wei92, Corollary 3.2] whose proof can again be taken verbatim.

Hence we obtain that for a ball complex $X$

$$
L_{n}\left(\mathbb{A}_{*}(X)\right) \cong H_{n}\left(X ; \mathbf{L}_{\bullet}(\mathbb{A})\right) \text { and } L^{n}\left(\mathbb{A}_{*}(X)\right) \cong H_{n}(X ; \mathbf{L} \bullet(\mathbb{A})) .
$$

We conclude the section by unravelling what it means to have an element in $L$ homology of a ball complex $X$ and of a pair of ball complexes $(X, A)$. It is, in fact, very similar to what we have when $X$ is a simplicial complex as explained in [Ran92, Example 12.9]. Hence an element $[(C, \psi)]$ in $L_{n}\left(\mathbb{A}_{*}(X)\right)$ is represented by a compatible collection of $(n-|\sigma|)$-dimensional quadratic Poincaré chain $(m-|\sigma|)$-ads

$$
\sigma \mapsto(C(\sigma), \psi(\sigma)),
$$

that means $(C(\sigma), \psi(\sigma)) \in \mathbf{L}_{n-m}^{(m-|\sigma|)}(\mathbb{A})$ for some $m \in \mathbb{Z}$. For a pair $(X, A)$ we obtain the same except we allow that $(C(\sigma), \psi(\sigma))$ is not necessarily Poincaré if $\sigma \in A$. This uses the localisation sequences in $L$-theory as in [Wei92, Theorem 2.3] or [Ran92, Proposition 13.11]. We will use notation $L_{n}\left(\mathbb{Z}_{*}(X, A)\right)$ in this context.

Remark 6.2. In the introduction we also used the notion of algebraic bordism categories. The precise definition is in [Ran92, Chapter 3]. Informally one considers not all quadratic complexes $(C, \psi)$ but only a subcategory $\mathbb{B} \subset \mathbb{B}(\mathbb{A})$ and the complexes do not have to be Poincaré, they may only be Poincaré up to a subcategory $\mathbb{C} \subset \mathbb{B}$ meaning the cofiber of the duality map has to be in $\mathbb{C}$.

The algebraic bordism category $\Lambda_{*}(X)$ is defined in [Ran92] and we have $\Lambda=$ $(\mathbb{A}, \mathbb{B}, \mathbb{C})$ in mind where $\mathbb{B}=\mathbb{B}(\mathbb{A})$ and $\mathbb{C} \subset \mathbb{B}$ is the subcategory of contractible complexes. With this notation we have $L_{n}\left(\mathbb{A}_{*}(X)\right)=L_{n}\left(\Lambda_{*}(X)\right)$. The symbol $\Lambda_{*}^{c}(X)$ means the same as $\Lambda_{*}(X)$ together with the assumption that the chain complexes are globally contractible (meaning contractible after the assembly [Ran92, Chapter 9]). The category $\Lambda^{*}(X)$ is defined just like $\Lambda_{*}(X)$ except that the underlying additive category with chain duality is $\mathbb{A}^{*}(X)$. We also mention the related category $\Lambda(X)$ from $\left[\right.$ Ran92] which consists of all chain complexes in $\mathbb{A}_{*}(X)$ which are only globally Poincaré. Its $L$-theory is not a homology theory.

For the sake of clarity we did not introduce connective versions of the algebraic bordism categories as in [Ran92, Chapter 15]. However, the proofs would work just as well in those cases. Similarly everything works also in the symmetric case.

\section{Signatures}

In this section we review how to obtain elements of various $L$-groups from geometric situations. This is a straightforward generalisation from simplicial complexes to structured cell complexes of what was done in [Ran92] and [KMM13]. The main idea is to make maps transverse to dual cells. This can be done since for a structured cell complex $X$ each dual cell $D(\sigma, X)$ has a trivial normal PL-bundle with the fibre given by the ball $\sigma$ itself. 
Let us first recall signatures over group rings. Let $F$ be a $k$-dimensional manifold with a reference map $r_{F}: F \rightarrow L$ to a structured cell complex $L$. The symmetric signature

$$
\operatorname{ssign}_{\pi_{1}(L)}(F) \in L^{k}\left(\mathbb{Z} \pi_{1}(L)\right)
$$

is represented by a symmetric chain complex

$$
(C, \varphi) \text { such that } C=C(F) \text { and } \varphi=r_{F}^{\%} \varphi_{F}[F],
$$

where $\varphi_{F}$ is the symmetric construction of [Ran80b, Section 1] or [Ran80b, Section 6$]$ in the relative case. Both are natural on the chain level.

Let $(f, b): M \rightarrow X$ be a degree one normal map from an $n$-dimensional manifold $M$ to an $n$-dimensional geometric Poincaré complex $X$ with a reference map $r_{X}: X \rightarrow K$ to a structured cell complex $K$. Let $U: \Sigma^{p} X_{+} \rightarrow \Sigma^{p} M_{+}$be an associated Umkehr map. The quadratic signature

$$
\operatorname{qsign}_{\pi_{1}(K)}(f, b) \in L_{n}\left(\mathbb{Z} \pi_{1}(K)\right)
$$

is represented by the quadratic chain complex

$$
(C, \psi) \text { such that } C=\mathcal{C}\left(f^{!}\right) \text {and } \psi=\left(r_{X}\right)_{\%} \psi_{U}[X]
$$

where $f^{!}$denotes the algebraic Umkehr map associated to $U$ and $\psi_{U}$ is the quadratic construction of [Ran80a, Section 1] or [Ran80b, Section 6].

Remark \%.1. We note that while the quadratic construction $\psi_{U}$ in $[\mathbf{R a n} 80 \mathrm{a}$, Sections 1,6] is described on the chain level, it is not known to be natural on the chain level (only in homology), see [Ran81, Chapter I, p. 30]. On the other hand we observe that a substitute property exists which is often sufficient. As noted in the above sources, the only problem preventing us from the naturality property is that there is no natural inverse to the suspension chain homotopy equivalence $C(X) \rightarrow \Sigma^{-p} C\left(\Sigma^{p}(X)\right)$. Suppose that $X$ is "dissected over" $K$ as in [Ran92, Example 9.12]. The suspension map respects the dissection and for each simplex $\sigma$ it restricts to the suspension map for $X[\sigma]$ and hence they individually have chain homotopy inverses. But now the argument from [Ran92, Proposition 4.7] tells us that there exists an inverse for $X$ which respects the dissection.

Let $F$ be a $k$-dimensional manifold with a reference map $r_{F}: F \rightarrow L$ which is a homotopy equivalence to a structured cell complex $L$. Such $r_{F}$ always exists for example using the fact that $F$ is an ENR. Make $r_{F}$ transverse to dual cells so that each $F(\sigma)=r_{F}^{-1}(D(\sigma, L))$ is a $(k-|\sigma|)$-dimensional manifold with boundary. A choice of the fundamental class $[F] \in C_{k}(F)$ projects to a choice of the fundamental class for each $C_{k-|\sigma|}(F(\sigma), \partial F(\sigma))$. By naturality of the symmetric construction on the chain level we obtain relative symmetric signatures for all $\sigma \in L$ which fit together to yield the symmetric signature over $L$

$$
\operatorname{ssign}_{L}(F) \in L^{k}\left(\mathbb{Z}_{*}(L)\right)
$$

represented by a symmetric chain complex over $L$

$$
(C, \varphi) \text { such that } C(\sigma)=C(F(\sigma), \partial F(\sigma)) \text { for } \sigma \in L,
$$

where $\varphi(\sigma)$ is the relative symmetric structure for $(F(\sigma), \partial F(\sigma))$ obtained as 
in [Ran80b, Section 6]. See [Ran92, Example 9.12], [KMM13, Definition 8.11] for details.

Let $(F, \partial F)$ be a $k$-dimensional manifold with boundary and with a reference map $r_{(F, \partial F)}:(F, \partial F) \rightarrow(L, \partial L)$ which is a homotopy equivalence of pairs to a pair of structured cell complexes $(L, \partial L)$. We obtain

$$
\operatorname{ssign}_{(L, \partial L)}(F, \partial F) \in L^{k}\left(\mathbb{Z}_{*}(L, \partial L)\right)
$$

again represented by a symmetric chain complex over $L$

$$
(C, \varphi) \text { such that } C(\sigma)=C(D(\sigma), \partial D(\sigma)) \text { for } \sigma \in L,
$$

where $\varphi(\sigma)$ is the relative symmetric structure for $(F(\sigma), \partial F(\sigma))$ obtained as in $[$ Ran80b, Section 6]. We note, however, that the chain complex $(C, \varphi)$ here is in general only Poincaré over balls outside $\partial L$.

Let $(f, b): M \rightarrow X$ be a degree one normal map between two $n$-dimensional manifolds with a reference map $r_{X}: X \rightarrow K$ which is a homotopy equivalence to a structured cell complex $K$ and such that both $r_{X}$ and $r_{X} \circ f$ are transverse to the dual cells of $K$, so that we have a compatible collection of degree one normal maps $(f(\sigma), b(\sigma)):(M(\sigma), \partial M(\sigma)) \rightarrow(X(\sigma), \partial X(\sigma))$. Let $U: \Sigma^{p} X_{+} \rightarrow \Sigma^{p} M_{+}$be an associated Umkehr map. It projects to Umkehr maps for all $\sigma \in K$. Using Remark 7.1 we obtain the relative quadratic signatures for each $\sigma \in K$ which fit together to yield a quadratic signature over $K$

$$
\operatorname{qsign}_{K}(f, b) \in L_{n}\left(\mathbb{Z}_{*}(K)\right)
$$

represented by the quadratic chain complex over $K$

$$
(C, \psi) \text { such that } C(\sigma)=\mathcal{C}\left(f^{!}(\sigma), \partial f^{!}(\sigma)\right) \text { for } \sigma \in K \text {. }
$$

Here $f(\sigma)=\left.f\right|_{\left(r_{X} \circ f\right)^{-1}(D(\sigma, L))}$, the notation $f^{!}(\sigma)$ denotes the algebraic Umkehr map obtained from the suitable projection of $U$ and $\psi(\sigma)$ is the resulting relative quadratic structure associated to the degree one normal map $(f(\sigma), b(\sigma))$ obtained as in [Ran80b, Section 6]. For details see [Ran92, Example 9.13], [KMM13, Definition 8.14].

Let $(f, b):(M, \partial M) \rightarrow(X, \partial X)$ be a degree one normal map between $n$-dimensional manifolds with boundary with a reference map $r_{(X, \partial X)}:(X, \partial X) \rightarrow(K, \partial K)$ which is a homotopy equivalence of pairs to a pair of structured cell complexes and such that both $r_{(X, \partial X)}$ and $r_{(X, \partial X)} \circ f$ are transverse to the dual cells of $K$. We obtain the relative quadratic signatures for each $\sigma \in K$ which fit together to yield a quadratic signature over $(K, \partial K)$

$$
\operatorname{qsign}_{(K, \partial K)}(f, b) \in L_{n}\left(\mathbb{Z}_{*}(K, \partial K)\right)
$$

represented by the quadratic chain complex over $K$

$$
(C, \psi) \text { such that } C(\sigma)=\mathcal{C}\left(f^{!}(\sigma), \partial f^{!}(\sigma)\right) \text { for } \quad \sigma \in K
$$

Again here $f(\sigma)=\left.f\right|_{\left(r_{X} \circ f\right)^{-1}(D(\sigma, L))}$, the notation $f^{!}(\sigma)$ denotes the algebraic Umkehr map obtained from the suitable projection of $U$ and $\psi(\sigma)$ is the resulting relative quadratic structure associated to the degree one normal map $(f(\sigma), b(\sigma))$ obtained as in $[\mathbf{R a n} 80 \mathbf{b}$, Section 6]. We note, however, that the chain complex $(C, \psi)$ here is in general only Poincaré over balls outside $\partial K$. 
Remark 7.2. Without much effort the signatures we presented here can also be generalised to the case when $X$ is a geometric Poincaré complex. The main difference is that when considering signatures over such $X$ one obtains elements of the $L$-theory of the category $\Lambda(X)$, see Remark 6.2, that means quadratic chain complexes dissected over $X$, which might only be Poincaré globally. See [KMM13, Section 14] for more details about signatures in these situations.

\section{Products}

Recall from [Ran80a, Section 8] that for rings $R$ and $S$ with involution we have natural products

$$
\begin{aligned}
& -\otimes-: L^{k}(R) \otimes L^{n}(S) \rightarrow L^{n+k}(R \otimes S), \\
& -\otimes-: L^{k}(R) \otimes L_{n}(S) \rightarrow L_{n+k}(R \otimes S),
\end{aligned}
$$

such that in both cases the underlying chain complex of the product is the usual tensor product of the underlying chain complexes

$$
\begin{aligned}
& \left(C, \varphi_{C}\right) \otimes\left(D, \varphi_{D}\right)=\left(C \otimes D, \varphi_{C} \otimes \varphi_{D}\right), \\
& \left(C, \varphi_{C}\right) \otimes\left(D, \psi_{D}\right)=\left(C \otimes D, \varphi_{C} \otimes \psi_{D}\right)
\end{aligned}
$$

and where $\varphi_{C} \otimes \varphi_{D}$ and $\varphi_{C} \otimes \psi_{D}$ are defined via a diagonal approximation of $W$ as in [Ran80a, p. 174]. Note that these definitions are again on the chain level.

Recall from [Ran80b, Section 8] that for maps $r_{F}: F \rightarrow B \pi, r_{F^{\prime}}: F^{\prime} \rightarrow B \pi^{\prime}$ and $(f, b): M \rightarrow X$ with $r_{X}: X \rightarrow B \pi^{\prime}$ these products satisfy product formulae

$$
\begin{aligned}
\operatorname{ssign}_{\mathbb{Z}\left[\pi \times \pi^{\prime}\right]}\left(F \times F^{\prime}\right) & =\operatorname{ssign}_{\mathbb{Z}[\pi]}(F) \otimes \operatorname{ssign}_{\mathbb{Z}\left[\pi^{\prime}\right]}\left(F^{\prime}\right), \\
\operatorname{qsign}_{\mathbb{Z}\left[\pi \times \pi^{\prime}\right]}\left(\operatorname{id}_{F} \times f, \operatorname{id}_{\nu_{F}} \times b\right) & =\operatorname{ssign}_{\mathbb{Z}[\pi]}(F) \otimes \operatorname{qsign}_{\mathbb{Z}\left[\pi^{\prime}\right]}(f, b) .
\end{aligned}
$$

In the symmetric case the proof uses that the acyclic models method induces a natural chain homotopy equivalence $C\left(F \times F^{\prime}\right) \rightarrow C(F) \otimes C\left(F^{\prime}\right)$ inverse to the cross product. The cross product produces a fundamental class cycle of $F \times F^{\prime}$ from the fundamental class cycles of $F$ and $F^{\prime}$ and the same argument as the one which shows the Cartan formula for Steenrod squares identifies the two symmetric structures. In the quadratic case the proof uses in addition that if a stable map $U: \Sigma^{p} X_{+} \rightarrow \Sigma^{p} M_{+}$ is a geometric Umkehr map for $(f, b)$ then id $\wedge U: \Sigma^{p}(F \times X)_{+} \rightarrow \Sigma^{p}(F \times M)_{+}$is a geometric Umkehr map for $(i d \times f$, id $\times b)$ and that the symmetric construction commutes naturally with suspensions. As before note that these arguments work in the relative case and on the chain level in the symmetric case and in the quadratic case we can use Remark 7.1.

The relative versions are straightforward. We emphasise that the above discussion says that the products are constructed naturally on the chain level in the symmetric case. In the quadratic case almost the same is true, use the substitute for naturality discussed in the previous section which allows us to say that we get the multiplicativity already on the chain level. The fact that the formulae in $[\mathbf{R a n} 80 \mathbf{b}]$ are stated in terms of $L$-groups is caused by the observation that the fundamental classes are only welldefined up to homology on the chain level.

Our aim in this section is to prove Theorem 1.1. We start with the construction of the products (1.2). 
Construction 8.1. We construct the products

$$
\begin{aligned}
& -\otimes-: L^{k}\left(\mathbb{Z}_{*}(L)\right) \otimes L^{n}\left(\mathbb{Z}_{*}(K)\right) \rightarrow L^{n+k}\left(\mathbb{Z}_{*}(L \times K)\right), \\
& -\otimes-: L^{k}\left(\mathbb{Z}_{*}(L)\right) \otimes L_{n}\left(\mathbb{Z}_{*}(K)\right) \rightarrow L_{n+k}\left(\mathbb{Z}_{*}(L \times K)\right)
\end{aligned}
$$

as a refinement of the products (8.1) defined via (8.2). Recall that an element in $L^{k}\left(\mathbb{Z}_{*}(L)\right)$ is represented by a $k$-dimensional symmetric chain complex $\left(C, \varphi_{C}\right)$ in $\mathbb{Z}_{*}(L)$. This means that it is an assignment $\sigma \mapsto(C(\sigma), \varphi(\sigma))$ where the value is an appropriate $(k-|\sigma|)$-dimensional symmetric Poincaré ad. Let similarly $\left(D, \varphi_{D}\right)$ represent an element in $L^{n}\left(\mathbb{Z}_{*}(K)\right)$ and $\left(D, \psi_{D}\right)$ represent an element in $L_{n}\left(\mathbb{Z}_{*}(K)\right)$. Define the products $(8.3)$ by the formulae

$$
\begin{aligned}
& \left(\left(C, \varphi_{C}\right),\left(D, \varphi_{D}\right)\right) \mapsto\left(\sigma \times \tau \mapsto\left(C(\sigma) \otimes D(\tau), \varphi_{C}(\sigma) \otimes \varphi_{D}(\tau)\right)\right), \\
& \left(\left(C, \varphi_{C}\right),\left(D, \psi_{D}\right)\right) \mapsto\left(\sigma \times \tau \mapsto\left(C(\sigma) \otimes D(\tau), \varphi_{C}(\sigma) \otimes \psi_{D}(\tau)\right)\right),
\end{aligned}
$$

where the products $\otimes$ on the right hand sides are the chain level products in (8.2). The products here are well defined, since products of Poincaré ads are Poincaré ads of correct dimensions, see (6.2). The products (1.2) are obtained from (8.3) via the isomorphisms (6.1).

Proof of Theorem 1.1. With all the work done so far, the proofs are quite straightforward due to the fact that the ball complex structure on a product of ball complexes is given by products of balls and that the dual cells are products of dual cells and that the products (8.4) actually come from products on the chain level.

Let $r_{F}: F \rightarrow L$ and $r_{F^{\prime}}: F^{\prime} \rightarrow K$ be transverse to dual cells and consider $\operatorname{ssign}_{L}(F)$ and $\operatorname{ssign}_{K}\left(F^{\prime}\right)$. These are represented by symmetric chain complexes over $L$ and $K$ respectively with underlying chain complexes such that for $\sigma \in L$ we have that $C(\sigma)=$ $C(D(\sigma, L), \partial D(\sigma, L))$ and for $\tau \in K$ we have that $D(\tau)=C(D(\tau, K), \partial D(\tau, K))$.

Now consider $r_{F} \times r_{F^{\prime}}: F \times F^{\prime} \rightarrow L \times K$. This map is already transverse to the dual cells $D(\sigma \times \tau, L \times K)=D(\sigma, L) \times D(\tau, K)$. Moreover, we have that $r_{F} \times r_{F^{\prime}}$ restricted to $\left(r_{F} \times r_{F^{\prime}}\right)^{-1}(D(\sigma \times \tau, L \times K))$ equals

$$
r_{F}\left|\times r_{F^{\prime}}\right|: F(\sigma) \times F^{\prime}(\tau) \rightarrow D(\sigma, L) \times D(\tau, K) .
$$

Hence from the multiplicativity of the relative products on the chain level we obtain the first desired formula. For the quadratic case let $(f, b): M \rightarrow X$ with $r_{X}: X \rightarrow K$ and $r_{F}: F \rightarrow L$ be transverse to the dual cells. Then both $r_{F} \times r_{X}$ and $\left(r_{F} \times r_{X}\right) \circ f$ are already transverse to the dual cells $D(\sigma \times \tau, L \times K)=D(\sigma, L) \times D(\tau, K)$ and the restriction maps are the product maps

$$
\left(\operatorname{id}_{F(\sigma)} \times f(\tau), \operatorname{id}_{\nu_{F(\sigma)}} \times b(\tau)\right): F(\sigma) \times M(\tau) \rightarrow F(\sigma) \times X(\tau) .
$$

Hence from the multiplicativity of the relative products on the chain level we obtain the second desired formula.

There are many relative versions of the products and product formulae above. They are obtained exactly as the absolute versions, except that the symmetric and quadratic complexes are only required to be Poincaré outside the boundaries. For simplicity we only discuss one of them which is also of interest for applications. 
Let $(F, \partial F)$ be a $k$-dimensional manifold with boundary and with a reference map $r_{(F, \partial F)}:(F, \partial F) \rightarrow(L, \partial L)$ which is a homotopy equivalence of pairs to a pair of structured cell complexes $(L, \partial L)$. In the previous section we constructed

$$
\operatorname{ssign}_{L, \partial L}(F, \partial F) \in L^{k}\left(\mathbb{Z}_{*}(L, \partial L)\right) .
$$

We have the relative product

$$
-\otimes-: L^{k}\left(\mathbb{Z}_{*}(L, \partial L)\right) \otimes L_{n}\left(\mathbb{Z}_{*}(K)\right) \rightarrow L_{n+k}\left(\mathbb{Z}_{*}(L \times K, \partial L \times K)\right)
$$

and again from the multiplicativity of the relative products on the chain level we obtain the product formula

$$
\operatorname{qsign}_{(L \times K, \partial L \times K)}\left(\operatorname{id}_{F} \times f, \operatorname{id}_{\nu_{F}} \times b\right)=\operatorname{ssign}_{(L, \partial L)}(F, \partial F) \otimes \operatorname{qsign}_{K}(f, b) .
$$

Let us now consider the special case when $(F, \partial F)=\left(D^{k}, S^{k-1}\right)$ and we take $r_{D^{k}}$ to be the identity and think of $D^{k}=[0,1]^{k}$ as a ball complex. Also recall that the $L$-groups over complexes are isomorphic to the homology groups with coefficients in the spectra $\mathbf{L}^{\bullet}=\mathbf{L}^{\bullet}(\mathbb{Z})$ and $\mathbf{L}_{\bullet}=\mathbf{L}_{\bullet}(\mathbb{Z})$. We observe that the product with

$$
\operatorname{ssign}_{\left(D^{k}, S^{k-1}\right)}\left(D^{k}, S^{k-1}\right) \in L^{k}\left(\mathbb{Z}_{*}\left(D^{k}, S^{k-1}\right)\right) \cong H_{k}\left(D^{k}, S^{k-1} ; \mathbf{L}^{\bullet}\right)
$$

commutes with the suspension isomorphism

$$
H_{n}\left(K ; \mathbf{L}_{\bullet}\right) \rightarrow H_{n+k}\left(D^{k} \times K, S^{k-1} \times K ; \mathbf{L}_{\bullet}\right)
$$

as follows.

We observe from the definitions that the Poincare duality isomorphism in symmetric $L$-theory sends

$$
\begin{gathered}
L^{k}\left(\mathbb{Z}_{*}\left(D^{k}, S^{k-1}\right)\right) \cong H_{k}\left(D^{k}, S^{k-1} ; \mathbf{L}^{\bullet}\right) \cong H^{0}\left(D^{k} ; \mathbf{L}^{\bullet}\right) \cong \pi_{0} \mathbf{L}^{\bullet} \\
\operatorname{ssign}_{\left(D^{k}, S^{k-1}\right)}\left(D^{k}, S^{k-1}\right) \mapsto 1
\end{gathered}
$$

and hence the symmetric signature $\operatorname{ssign}_{\left(D^{k}, S^{k-1}\right)}\left(D^{k}, S^{k-1}\right)$ is, in fact, the fundamental class of $\left(D^{k}, S^{k-1}\right)$ in the homology groups with coefficients in the symmetric $L$-theory spectrum $\mathbf{L}^{\bullet}$. Now the general theory of products in ring spectra and module spectra over ring spectra as in [Ada74] tells us that the product with the fundamental class of $\left(D^{k}, S^{k-1}\right)$ gives the suspension isomorphism. There are also versions with appropriate connective $L$-spectra, see [Ran92, Chapter 15] if needed.

Remark 8.2. Without much effort the products and the product formulae we obtained here can be extended to a situation when $K$ and $L$ are geometric Poincaré complexes. The main difference is that, as noted above in Remark 7.2, the signature lands in the $L$-theories of algebraic bordism categories of chain complexes which are only globally Poincaré. We leave the details for the reader.

\section{References}

[Ada74] J.F. Adams. Stable Homotopy and Generalised Homology. Chicago Lectures in Mathematics. University of Chicago Press, Chicago, Ill., 1974.

[AF12] S. Adams-Florou. Homeomorphisms, homotopy equivalences and chain complexes. arXiv:1205.3024, May 2012. 
[BLM14] M. Banagl, G. Laures, and J.E. McClure. The L-homology fundamental class for IP-spaces and the stratified Novikov conjecture arXiv:1404.5395, 2014.

[BRS76] S. Buoncristiano, C.P. Rourke, and B.J. Sanderson. A Geometric Approach to Homology Theory, volume 18 of London Mathematical Society Lecture Note Series. Cambridge University Press, Cambridge, New York, Melbourne, 1976.

[KMM13] P. Kühl, T. Macko, and A. Mole. The total surgery obstruction revisited. Münster J. Math., 6(1):181-269, 2013.

[LM14] G. Laures and J.E. McClure. Multiplicative properties of Quinn spectra. Forum Math., 26(4):1117-1185, 2014.

[LR92] W. Lück and A.A. Ranicki. Surgery obstructions of fibre bundles. J. Pure Appl. Algebra, 81(2):139-189, 1992.

[McC75] C. McCrory. Cone complexes and PL transversality. Trans. Amer. Math. Soc., 207:269-291, 1975.

[Ran79] A.A. Ranicki. The total surgery obstruction. In Algebraic Topology, Aarhus 1978 (Proc. Sympos., Univ. Aarhus, Aarhus, 1978), volume 763 of Lecture Notes in Mathematics, pages 275-316. Springer, Berlin, 1979.

[Ran80a] A.A. Ranicki. The algebraic theory of surgery. I. Foundations. Proc. Lond. Math. Soc. (3), 40(1):87-192, 1980.

[Ran80b] A.A. Ranicki. The algebraic theory of surgery. II. Applications to topology. Proc. Lond. Math. Soc. (3), 40(2):193-283, 1980.

[Ran81] A.A. Ranicki. Exact Sequences in the Algebraic Theory of Surgery, volume 26 of Mathematical Notes. Princeton University Press, Princeton, N.J., 1981.

[Ran92] A.A. Ranicki. Algebraic L-Theory and Topological Manifolds, volume 102 of Cambridge Tracts in Mathematics. Cambridge University Press, Cambridge, 1992.

[Wei92] M. Weiss. Visible L-theory. Forum Math., 4(4):465-498, 1992.

[Whi78] G.W. Whitehead. Elements of Homotopy Theory, volume 61 of Graduate Texts in Mathematics. Springer-Verlag, New York, 1978.

Spiros Adams-Florou Spiros.Adams-Florou@glasgow.ac.uk

The Mathematics and Statistics Building, University of Glasgow, University Place, Glasgow G12 8QQ, United Kingdom

Tibor Macko macko@mat.savba.sk

Mathematical Institute, Slovak Academy of Sciences, Štefánikova 49, SK-814 73

Bratislava, Slovakia 